JOURNAL OF THE

AMERICAN MATHEMATICAL SOCIETY

Volume 17, Number 4, Pages 871-908

S 0894-0347(04)00461-8

Article electronically published on August 17, 2004

\title{
VEECH SURFACES AND COMPLETE PERIODICITY IN GENUS TWO
}

\author{
KARIANE CALTA
}

\section{INTRODUCTION}

A translation surface is a pair $(M, \omega)$ where $M$ is a closed Riemann surface and $\omega$ is an Abelian differential on $M$. Away from the zeros of $\omega$, a chart $z$ can be chosen so that $\omega=d z$, and this determines a Euclidean metric in that chart. The change of coordinates away from the zeros of $\omega$ are of the form $z \rightarrow z+c$. In the neighborhood of a zero, one can choose a coordinate $z$ so that $\omega=z^{k} d z$. The total angle about this zero is $2(k+1) \pi$ and the order of the zero is $k$. Such a zero is called a conical singularity. As a result of this definition, a translation surface can also be thought of as a two-manifold with transition functions which are translations and which has a finite number of conical singularities, arising from the zeros of $\omega$. Each of these singularities has total angle $2 \pi n$ for $n \in \mathbb{Z}^{+}$. Thus, one can also think of a translation surface as a finite number of polygons in the plane $\mathbb{R}^{2}$, glued along parallel sides. Throughout, we will often denote a translation surface by $S$, and this notation should be thought of as incorporating both the Riemann surface $M$ and the differential $\omega$.

If $S$ is a translation surface, a saddle connection is a geodesic connecting two (not necessarily distinct) conical singularities of $S$ which has no singularities in its interior. Note that since the metric on $S$ is Euclidean away from the zeros of $\omega$, saddle connections are straight lines, but in general, geodesics which pass through singularities are unions of saddle connections where the line segments comprising the geodesic are allowed to switch directions at the conical singularities of $S$. A closed geodesic on $S$ which does not pass through any singularities determines a cylinder of parallel freely homotopic closed curves such that the boundaries of the cylinder consist of unions of saddle connections. A direction $v$ on $S$ is said to be completely periodic if all the trajectories in the direction $v$ are either closed or begin and end at a singularity. In other words, $v$ is completely periodic if in the direction $v$, the surface decomposes as a union of cylinders. A surface $S$ is completely periodic if any direction in which there is at least one cylinder of closed trajectories is completely periodic.

It is well known that if a translation surface $S$ has genus $g \geq 1$, then the number of zeros of $\omega$, counting multiplicity, is $2 g-2$. Thus if the multiplicities of the $k$ zeros of $\omega$ are given by $\alpha_{1}, \cdots, \alpha_{k}$, then $\alpha_{1}+\cdots+\alpha_{k}=2 g-2:=\alpha$. That is, $\left\{\alpha_{1}, \cdots, \alpha_{k}\right\}$ is a partition of $\alpha$. Now, fix a genus $g$ and a partition $\alpha$ of $2 g-2$. We let $\mathcal{H}(\alpha)$ denote the space of translation surfaces $(M, \omega)$ where $M$ is a Riemann

Received by the editors January 13, 2003.

1991 Mathematics Subject Classification. Primary 37A99; Secondary 37E15, 37D40, 37D50.

(C)2004 American Mathematical Society 
surface of genus $g$ and $\omega$ is an Abelian differential on $M$ whose zeros correspond in the way described above to the partition $\alpha$. For example, the set of translation surfaces of genus two is stratified as $\mathcal{H}(1,1) \sqcup \mathcal{H}(2)$, where in $\mathcal{H}(1,1)$ the 1 -form has two distinct simple zeros, and in $\mathcal{H}(2)$ it has a single double zero.

There is an action of the group $S L(2, \mathbb{R})$ on each stratum $\mathcal{H}(\alpha)$. If we think of a translation surface $S$ as a two-manifold with transition functions which are translations and which has a finite number of conical singularities, and if $g \in$ $S L(2, \mathbb{R})$, then $g S$ is the two-manifold which has charts that are given by $g \circ f_{i}$ where $f_{i}$ is a chart of $S$. It is easily checked that the transition functions of $g S$ are still translations and the number of singularities and their total angles are preserved. Alternatively, if $S=P_{1} \cup \ldots \cup P_{n}$, where each $P_{i}$ is a polygon in the plane $\mathbb{R}^{2}$, then $g S=g P_{1} \cup \ldots \cup g P_{n}$. A translation surface is said to be Veech if its stabilizer under this action of $S L(2, \mathbb{R})$ is a lattice. It is a theorem of Veech that Veech surfaces are completely periodic [V1], but the converse is not true. See Section 6 for an example of a surface in $\mathcal{H}(1,1)$ which is completely periodic but not Veech.

For a curve $\gamma \subset S$, let $p(\gamma)$ denote $\int_{\gamma}(\omega)$, where $\omega$ is the 1-form defining the translation surface structure. We identify $\mathbb{R}^{2}$ with $\mathbb{C}$. Define a translation surface $S$ to be quadratic if there exists $d>0$ (not a perfect square) such that $p\left(H_{1}(S, \mathbb{Z})\right) \subset$ $\mathbb{Q}(\sqrt{d}) \times \mathbb{Q}(\sqrt{d})$. Any Veech surface in genus two (perhaps after rescaling) is known to be quadratic. This is proved rather indirectly in Theorem 4.3 but has also been attributed to Thurston (unpublished).

Let $C$ be a cylinder of periodic trajectories in the direction $v$. We use the terms width for the length of the periodic trajectory and height for the distance across the cylinder in the orthogonal direction. If $v^{\prime}$ is any other direction, we can talk about the twist of $C$ along $v^{\prime}$. First suppose the cylinder is bounded on each side by a single saddle connection. Then we can realize the cylinder, via the map $p$, as a parallelogram in the plane $\mathbb{R}^{2}$ with singularities at each of its vertices. Without loss of generality, assume the periodic trajectories are horizontal, and place the bottom left corner of the cylinder at the origin. Then the twist in the direction $v^{\prime}$, where the angle between $v^{\prime}$ and the horizontal is between 0 and $\pi / 2$, is computed in the following way. Starting at the origin, travel up the cylinder along the direction $v^{\prime}$ to the top edge of the cylinder and call the point of intersection $z$. The twist along $v^{\prime}$ is the horizontal distance of the path from $z$ to the singularity on the top edge (moving to the right along the top of the cylinder). Note that by cutting and pasting, we can always assume that the angle between $v^{\prime}$ and the horizontal is between 0 and $\pi / 2$ and that the twist is between 0 and the width of the cylinder. If a cylinder has more than one saddle connection on each boundary, then there is a choice for the twist since if we realize the cylinder as a parallelogram in the plane, it will have more marked points, corresponding to the singularities, along the boundaries than just those at the corners. We will choose the twist so that if some pair of saddle connections of the same length lie directly above each other in the cylinder in the direction in which we are measuring the twist, then the twist in this direction is zero. With this convention, the twist satisfies our equations (1.2) and (1.4) in the statements of the theorems below.

Note that with these definitions, if $\alpha_{i}$ is the closed core curve of a cylinder $C_{i}$, then $\int_{\alpha_{i}} \omega$ is an absolute period for the 1 -form $\omega$. On the other hand, if $\gamma$ is a saddle connection joining two distinct singularities, then $\int_{\gamma} \omega$ is a relative period for $\omega$. 

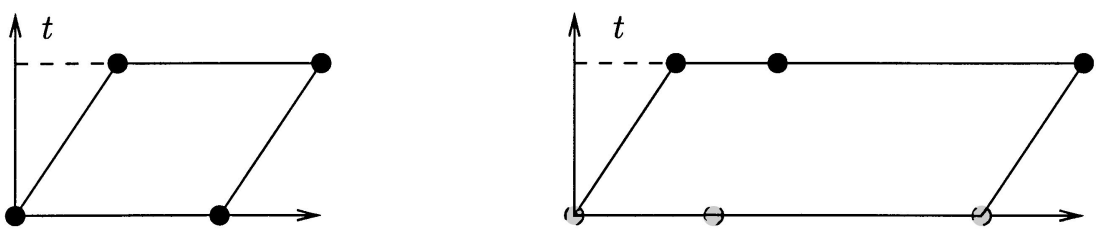

FiguRE 1. The twists in the vertical direction of two horizontal cylinders

Our main results are a classification of all Veech surfaces in $\mathcal{H}(2)$ and a classification of all completely periodic surfaces in genus two, excluding in both instances those surfaces which are torus covers and thus represent elementary cases. We do not consider one-cylinder decompositions in the theorems below since these lead to torus covers. The interested reader may find a nice treatment of torus covers in G.J.

Theorem 1.1. Let $S$ be a Veech surface in $\mathcal{H}(2)$ which cannot be rescaled so that $p\left(H_{1}(S, \mathbb{Z})\right) \subset \mathbb{Q} \times \mathbb{Q}$. Let $v$ be a completely periodic direction for $S$. Suppose the cylinder decompositon in the direction $v$ has two cylinders. Let $w_{1}, w_{2}, h_{1}, h_{2}, t_{1}, t_{2}$ be the widths, heights and twists of these two cylinders. The twists are measured along some direction $v^{\prime}$ which we assume is also completely periodic. After rescaling the surface, we may assume that these variables are algebraic integers in $\mathbb{Q}(\sqrt{d})$ where $d$ is some square-free, positive integer. Then the following equations are satisfied:

$$
\begin{aligned}
w_{1} \bar{h}_{1} & =-w_{2} \bar{h}_{2}, \\
\bar{w}_{1} t_{1}+\bar{w}_{2} t_{2} & =w_{1} \bar{t}_{1}+w_{2} \overline{t_{2}}, \quad 0 \leq t_{i}<w_{i}
\end{aligned}
$$

(where the bar denotes conjugation in $\mathbb{Q}(\sqrt{d})$.)

Conversely, let $S$ be a surface in $\mathcal{H}(2)$. Suppose there exists a direction $v$ in which there is a cylinder decomposition such that the heights, widths and twists (along some completely periodic direction $\left.v^{\prime}\right)$ belong to $\mathbb{Q}(\sqrt{d})$ and satisfy (1.1) and (1.2). Then $S$ is Veech.

We note that a different classification of Veech surfaces in genus two, formulated in terms of Jacobians with real multiplication, was obtained by C. McMullen in [M1].

In order to state the classification of all completely periodic surfaces in genus two, we first establish some notation for surfaces in the stratum $\mathcal{H}(1,1)$. Suppose $v$ is a completely periodic direction for $S \in \mathcal{H}(1,1)$. Without loss of generality, we may assume that in the direction $v, S$ decomposes into three cylinders, and for $1 \leq i \leq 3$, let $w_{i}, h_{i}$ and $t_{i}$ denote the widths, heights and twists. After renumbering, we may assume that $w_{3}=w_{1}+w_{2}$. Define $s_{1}=h_{1}+h_{3}, s_{2}=h_{2}+h_{3}, \tau_{1}=t_{1}+t_{3}$, $\tau_{2}=t_{2}+t_{3}$. Let $\mathcal{O}_{d}^{+}$denote the positive algebraic integers in $\mathbb{Q}(\sqrt{d})$. Consider the following equations:

$$
\begin{aligned}
w_{1} \bar{s}_{1} & =-w_{2} \bar{s}_{2}, \\
\bar{w}_{1} \tau_{1}+\bar{w}_{2} \tau_{2} & =w_{1} \bar{\tau}_{1}+w_{2} \bar{\tau}_{2}, \quad 0 \leq \tau_{i}<w_{i}+w_{3} .
\end{aligned}
$$

Theorem 1.2. In $\mathcal{H}(2)$ every completely periodic surface is Veech. In $\mathcal{H}(1,1)$, if a surface has a cylinder decomposition in some direction $v$ such that the heights, 
widths and twists satisfy (1.3) and (1.4), then it is completely periodic. Conversely, if $S \in \mathcal{H}(1,1)$ is completely periodic, then after rescaling, either $p\left(H_{1}(S)\right) \subset \mathbb{Q} \times \mathbb{Q}$ or $p\left(H_{1}(S)\right) \subset \mathbb{Q}(\sqrt{d}) \times \mathbb{Q}(\sqrt{d})$ for some square-free $d>0$. If $S$ cannot be rescaled so that $p\left(H_{1}(S)\right) \subset \mathbb{Q} \times \mathbb{Q}$ and if $v$ is any completely periodic direction, then (1.3) and (1.4) hold, where the $w_{i}, s_{i}, \tau_{i}$ denote the parameters described above of the cylinder decomposition along $v$ and the twists are measured along another completely periodic direction $v^{\prime}$.

The proofs of both of these theorems rely upon information which can be obtained from the $J$-invariant of translation surfaces defined by Kenyon and Smillie in [KS. In fact, (1.1) and (1.2) arise from this invariant. Thus we begin Section 2 by recalling the definition of the $J$-invariant and by computing the invariant for certain types of surfaces in $\mathcal{H}(2)$ and $\mathcal{H}(1,1)$. In this section, and for the rest of the paper, we will examine two-cylinder decompositions of surfaces in $\mathcal{H}(2)$ and twoor three-cylinder decompositions of surfaces in $\mathcal{H}(1,1)$. The one-cylinder decompositions are handled separately in the Appendix. Section 3 includes a discussion of a particular class of maps known as interval exchange transformations which are simply orientation-preserving piecewise isometries of an interval. If a surface $S$ has an oriented measured foliation, then an interval $I$ transverse to the foliation determines an interval exchange transformation which is the first return map to $I$ under the flow in the direction of the foliation. Since a cylinder decomposition of a surface describes a foliation, the complete periodicity of a direction $v$ is equivalent to the periodicity of the interval exchange transformation which arises as the first return map for an interval transverse to the direction $v$. In Section 4 , we define a certain Property $X$ of translation surfaces which will act as an intermediate step in the proofs of the classification theorems. In this section, we will tie together several lemmas from Sections 2 and 3 in order to prove, for example, that in genus two, the notion of Property $X$ is equivalent to the notion of the complete periodicity of a surface. Finally, using the results of Section 4, in Section 5 we classify Veech surfaces in $\mathcal{H}(2)$ and in Section 6, the completely periodic surfaces in genus two. Furthermore, in Section 6, we find certain closed $S L(2, \mathbb{R})$-invariant submanifolds in the stratum $\mathcal{H}(1,1)$, which were also found by McMullen in M1]. The finding is significant because heretofore the only known closed, invariant submanifolds of the moduli space of Abelian differentials were the $S L(2, \mathbb{R})$ orbits of Veech surfaces $\mathrm{V} 2$, the strata themselves, and those obtained by a ramified covering construction. A classification of the closed, invariant submanifolds within a stratum $\mathcal{H}(\alpha)$ would be a first step in proving a version of Ratner's theorem $\mathrm{R}$ for the given stratum. Ratner's theorem is the classification of the measures invariant under the action of a unipotent subgroup on a homogeneous space $H / \Gamma$, where $H$ is a Lie group and $\Gamma$ a lattice in $H$. Thus, one can ask if a similar theorem holds for the unipotent action on a stratum, restricted to the $S L(2, \mathbb{R})$-invariant submanifolds of the stratum. In the work M2], McMullen determined the closed, invariant submanifolds in the genus two strata and classified the ergodic $S L(2, \mathbb{R})$-invariant measures.

\section{The $J$-INVARIANT}

The proofs of the results stated in this paper use the $J$-invariant for translation surfaces defined by Kenyon and Smillie in their paper [KS]. We recall the definition here. If $P$ is a polygon in $\mathbb{R}^{2}$ with vertices $v_{1}, \ldots, v_{n}$ in counterclockwise order 
about the boundary of $P$, then

$$
J(P)=v_{1} \wedge v_{2}+\ldots+v_{n-1} \wedge v_{n}+v_{n} \wedge v_{1}
$$

where $\wedge$ is taken to mean $\wedge_{\mathbb{Q}}$ and $\mathbb{R}^{2}$ is viewed as a $\mathbb{Q}$-vector space. A straightforward calculation yields the following theorem.

Theorem 2.1. $J(P)$ is translation invariant. That is, if $v \in \mathbb{R}^{2}$ and if the translation of $P$ by the vector $v$ is denoted by $P+v$, then $J(P)=J(P+v)$.

If $S$ is a translation surface with a cellular decomposition into planar polygons $P_{1} \cup \ldots \cup P_{k}, J(S)=\sum_{i=1}^{k} J\left(P_{i}\right)$. It is shown in [KS] that $J$ is independent of the decomposition, which justifies the notion of the $J$-invariant. Note, however, that if a polygon $P^{\prime}$ is obtained from a polygon $P$ by adding an additional marked point (to be thought of as another vertex) on one of the sides of $P$, then in general $J(P) \neq J\left(P^{\prime}\right)$. Using this definition, we can calculate the $J$-invariant of certain genus two translation surfaces.

Lemma 2.1. The J-invariant for a parallelogram $Q$ with vertices $(0,0),(w, 0)$, $(t+w, h)$, and $(t, h)$, listed in counterclockwise order, is given by

$$
J(Q)=2\left(\left(\begin{array}{l}
w \\
0
\end{array}\right) \wedge\left(\begin{array}{l}
t \\
h
\end{array}\right)\right) .
$$

Proof. Since $Q$ has vertices $(0,0),(w, 0),(t+w, h)$, and $(t, h)$, listed in counterclockwise order, the definition of $J$ implies

$$
\begin{aligned}
J(Q) & =\left(\begin{array}{l}
0 \\
0
\end{array}\right) \wedge\left(\begin{array}{l}
w \\
0
\end{array}\right)+\left(\begin{array}{l}
w \\
0
\end{array}\right) \wedge\left(\begin{array}{c}
w+t \\
h
\end{array}\right)+\left(\begin{array}{c}
w+t \\
h
\end{array}\right) \wedge\left(\begin{array}{l}
t \\
h
\end{array}\right)+\left(\begin{array}{l}
t \\
h
\end{array}\right) \wedge\left(\begin{array}{l}
0 \\
0
\end{array}\right) \\
& =2\left(\left(\begin{array}{l}
w \\
0
\end{array}\right) \wedge\left(\begin{array}{l}
t \\
h
\end{array}\right)\right) .
\end{aligned}
$$

Lemma 2.2. Suppose $S \in \mathcal{H}(2)$ has a two-cylinder decomposition in the horizontal direction as in Figure 2. Then the J-invariant for $S$ is

$$
J(S)=2\left(\left(\begin{array}{c}
w_{1} \\
0
\end{array}\right) \wedge\left(\begin{array}{c}
t_{1} \\
h_{1}
\end{array}\right)+\left(\begin{array}{c}
w_{2} \\
0
\end{array}\right) \wedge\left(\begin{array}{c}
t_{2} \\
h_{2}
\end{array}\right)\right)
$$

where $w_{i}, h_{i}, t_{i}$ are the width, height and twist parameters for the two cylinders. If $S \in \mathcal{H}(1,1)$ has a two- or three-cylinder decomposition in the horizontal direction as in Figure 3 , then the J-invariant is given by

$$
J(S)=2\left(\left(\begin{array}{c}
w_{1} \\
0
\end{array}\right) \wedge\left(\begin{array}{c}
\tau_{1} \\
s_{1}
\end{array}\right)+\left(\begin{array}{c}
w_{2} \\
0
\end{array}\right) \wedge\left(\begin{array}{c}
\tau_{2} \\
s_{2}
\end{array}\right)\right)
$$

where the cylinders are numbered so that $w_{3}=w_{2}+w_{1}$, and we define $\tau_{i}=t_{i}+t_{3}$, and $s_{i}=h_{i}+h_{3}$ for $i=1,2$.

Proof. See the Appendix. 


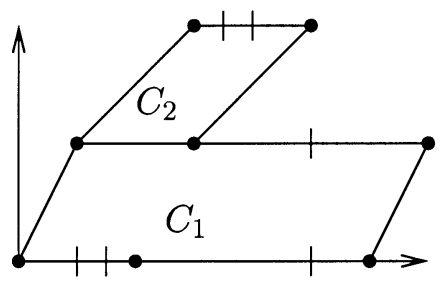

Figure 2. A two-cylinder decomposition of a surface $S \in \mathcal{H}(2)$

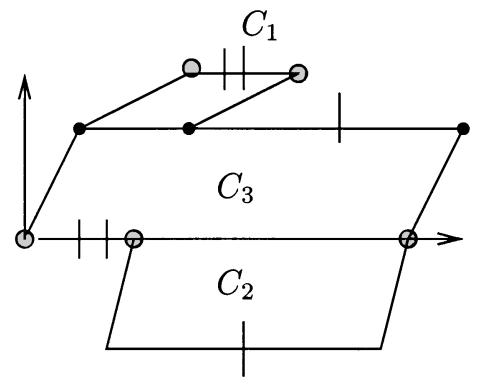

Figure 3. A three-cylinder decomposition of a surface $S \in \mathcal{H}(1,1)$

In addition to the $J$-invariant, Kenyon and Smillie define two linear projections $J_{x x}, J_{y y}: \mathbb{R}^{2} \wedge_{\mathbb{Q}} \mathbb{R}^{2} \rightarrow \mathbb{R} \wedge_{\mathbb{Q}} \mathbb{R}$ given on basis elements as

$$
\begin{aligned}
& J_{x x}\left(\left(\begin{array}{l}
a \\
b
\end{array}\right) \wedge\left(\begin{array}{l}
c \\
d
\end{array}\right)\right)=a \wedge c, \\
& J_{y y}\left(\left(\begin{array}{l}
a \\
b
\end{array}\right) \wedge\left(\begin{array}{l}
c \\
d
\end{array}\right)\right)=b \wedge d
\end{aligned}
$$

and one linear projection $J_{x y}: \mathbb{R}^{2} \wedge_{\mathbb{Q}} \mathbb{R}^{2} \rightarrow \mathbb{R} \otimes_{\mathbb{Q}} \mathbb{R}$ defined on basis elements as

$$
J_{x y}\left(\left(\begin{array}{l}
a \\
b
\end{array}\right) \wedge\left(\begin{array}{l}
c \\
d
\end{array}\right)\right)=a \otimes d-c \otimes b .
$$

Given this last definition, we have the following corollary of Lemma 2.2

Corollary 2.3. Let $S$ be a genus two translation surface which has a horizontal cylinder decomposition with width and height parameters $w_{i}$ and $h_{i}$. Then

$$
J_{x y}(S)=2 \sum_{i=1}^{n} w_{i} \otimes h_{i}
$$

where $n=1,2$ or 3 is the number of cylinders in the decomposition.

Kenyon and Smillie prove the following theorem in [KS], which we will use repeatedly, regarding the projections of $J$.

Theorem 2.2. Suppose that the horizontal direction $x$ on a translation surface $S$ is completely periodic, i.e., decomposes as a union of periodic trajectories bounded by saddle connections. Then $J_{y y}(S)=0$. 
Building upon the notions of $J_{y y}(S)$ and $J_{x x}(S)$, we will define $J_{v v}(S)$ where $v$ is an arbitrary direction on a translation surface $S$. First, if $v$ is either the horizontal or vertical direction, $J_{v v}(S)$ has already been defined, and we will retain this definition. So suppose that $v$ is neither the horizontal nor vertical direction, and let the vector $\left(\begin{array}{l}1 \\ q\end{array}\right)$ represent the direction $v$. If $g_{q} \in S L(2, \mathbb{R})$ is given by

$$
g_{q}=\left(\begin{array}{cc}
1 & 0 \\
-q & 1
\end{array}\right)
$$

so that $g_{q}\left(\begin{array}{l}1 \\ q\end{array}\right)=\left(\begin{array}{l}1 \\ 0\end{array}\right)$, then we define $J_{v v}(S)=J_{y y}\left(g_{q} S\right)$. We have the following lemma regarding the choice of matrix $g$ such that $g\left(\begin{array}{l}1 \\ q\end{array}\right)=\left(\begin{array}{l}1 \\ 0\end{array}\right)$.

Lemma 2.4. Let the direction $v$ on the translation surface $S$ be represented by the vector $\left(\begin{array}{l}1 \\ q\end{array}\right)$, where $v$ is neither the horizontal nor vertical direction, and suppose that $g \in S L(2, \mathbb{R})$ is any matrix such that $g\left(\begin{array}{l}1 \\ q\end{array}\right)=\left(\begin{array}{l}1 \\ 0\end{array}\right)$. Then $J_{y y}(g S)=J_{y y}\left(g_{q} S\right)$.

Proof. See the Appendix.

Given a pair of linearly independent directions $v, w$ on $S$, let $v^{\prime}, w^{\prime}$ be vectors in the directions $v$ and $w$ so that there exists an element $g \in S L(2, \mathbb{R})$ with $g v^{\prime}=\left(\begin{array}{l}1 \\ 0\end{array}\right)$ and $g w^{\prime}=\left(\begin{array}{l}0 \\ 1\end{array}\right)$ and define $J_{v w}(S)=J_{x y}(g S)$. (In the case that $v=\left(\begin{array}{l}0 \\ 1\end{array}\right)$ and $w=\left(\begin{array}{l}1 \\ 0\end{array}\right)$, define $J_{v w}(S)=J_{y x}(S)$ where $J_{y x}\left(\left(\begin{array}{l}a \\ b\end{array}\right) \wedge\left(\begin{array}{l}c \\ d\end{array}\right)\right)=b \otimes c-d \otimes a$.) It can be shown that a different choice of $v^{\prime}, w^{\prime}$ will multiply each of the components of each of the tensors comprising $J_{v w}$ by the same number.

An alternative formula for the $J$-invariant, proven by Arnoux in [A], is

$$
J=2 \sum_{i=1}^{g} p\left(a_{i}\right) \wedge p\left(b_{i}\right)
$$

where $\left\{a_{i}, b_{i}\right\}$ is a symplectic homology basis for the surface $S$.

\section{INTERVAL EXCHANGE TRANSFORMATIONS}

In this section, we recall the definition of an interval exchange transformation and discuss an important relationship between interval exchange transformations and foliations of translation surfaces which we will use to show in Section 4 that any genus two surface having Property X must be completely periodic.

An interval exchange transformation or iet is an orientation-preserving piecewise isometry of an interval $[a, b]$. In other words, an iet acts by translations on subintervals of $[a, b]$ with a finite number of points of discontinuity. Formally, suppose that $I$ and $I^{\prime}$ are two copies of this interval, and let $a=\lambda_{0}<\lambda_{1}<\cdots<\lambda_{n}=b$ and $a=\lambda_{0}{ }^{\prime}<\lambda_{1}{ }^{\prime}<\cdots<\lambda_{n}{ }^{\prime}=b$ be partitions of the intervals $I$ and $I^{\prime}$, respectively. Furthermore, let $I_{j}=\left[\lambda_{j-1}, \lambda_{j}\right]$ and $I_{j}{ }^{\prime}=\left[\lambda_{j-1}{ }^{\prime}, \lambda_{j}{ }^{\prime}\right]$ for $1 \leq j \leq n$. Let $\sigma$ be a permutation of the set $\{1, \cdots, n\}$ and assume that the length $l_{j}$ of $I_{j}$ is equal to the length $l_{\sigma(j)}{ }^{\prime}$ of $I_{\sigma(j)}{ }^{\prime}$. Then we define $f:[a, b] \rightarrow[a, b]$ outside of the set $\left\{\lambda_{0}, \cdots, \lambda_{n}\right\}$ as $f(x)=x+\tau_{j}$ where $x \in \stackrel{\circ}{I_{j}}$ for some $1 \leq j \leq n$, and $\tau_{j}=\lambda_{\sigma(j)}{ }^{\prime}-\lambda_{j}$ is the amount the interval $\stackrel{\circ}{I}_{j}$ is translated by $f$. Then $f$ is an interval exchange transformation on $n$ intervals.

A measured, oriented foliation of a surface $S$ is given by a closed 1-form $\rho$ such that for any transversal $\tau: I \rightarrow S, I=[0,1]$, there exist coordinates on $S$ so that $\tau^{*} \rho=d x$. One can define a first return map $f: I \rightarrow I$ so that $\tau(f(x))$ is the point 
where the leaf through $\tau(x)$ again crosses $\tau(I)$. This can be found, for example, in [S] and implies the following theorem.

Theorem 3.1. On a translation surface $S$, the flow in the direction $v$ partitions $S$ into domains which are invariant under the flow, each of which is either a cylinder of periodic trajectories parallel to $v$ or on which the flow is minimal. The number of domains is bounded by a constant which depends on the genus of the surface but not on the direction.

Since our classification theorems involve genus two surfaces in particular, we will make use of the following corollary of Theorem 3.1

Corollary 3.1. Let $v$ be a direction on a genus two translation surface $S$. Suppose $I$ is a transverse interval and that the trajectories emitted from the endpoints of I in the positive direction hit a singularity of $S$ before returning to I. Let $f_{I}$ denote the first return map to $I$. If $S \in \mathcal{H}(2)$, then $f_{I}$ exchanges at most four subintervals, and if $S \in \mathcal{H}(1,1)$, it exchanges at most five subintervals.

Proof. First, suppose that $S \in \mathcal{H}(2)$. Then $S$ has one singularity of total angle $6 \pi$ and hence at most three separatrices emanating from the singularity in any given direction $v$. If $I$ is a transverse interval which satisfies the conditions described in the statement of the corollary, then $I$ has at most four subintervals associated to the first return map $f_{I}$. To see this, note that if the endpoints of $I$ are distinct, there is at most one point in the interior of $I$ whose trajectory hits the singularity and does not return to $I$ in addition to the at most two points of $I$ which are mapped to the endpoints of $I$ and the endpoints of $I$ themselves. These are the partition points associated to $f_{I}$. If the endpoints of $I$ coincide, then there are at most two interior points of $I$ whose trajectories hit the singularity before returning and at most one interior point which is mapped to the endpoint. In either case, $f_{I}$ exchanges at most four subintervals.

Now suppose $S \in \mathcal{H}(1,1)$. Since each singularity has total angle $4 \pi$, there can be at most two separatrices emanating from each singularity in any given direction. If the endpoints of $I$ do not coincide, there are at most two interior points of $I$ whose trajectories hit the singularity and do not return to $I$ and at most two points of $I$ which are mapped to the endpoints of $I$ by the first return map $f_{I}$. If the endpoints do coincide, there are at most three interior points of $I$ whose trajectories hit the singularity before returning to $I$ and at most one interior point which is sent to the endpoint of $I$. Again, $f_{I}$ exchanges at most five subintervals.

We will also make use of the SAF invariant of an interval exchange transformation $f$. With notation as above, we have the definition (see [KS])

$$
S A F(f)=\sum_{j=1}^{n} l_{j} \wedge \tau_{j}
$$

The following results concerning the SAF invariant defined by an interval exchange transformation are proved by Arnoux in [A].

Theorem 3.2 (Arnoux). If $f$ is a periodic interval exchange transformation, then $S A F(f)=0$.

Lemma 3.2 (Arnoux). Let $f$ be an interval exchange transformation on the in-

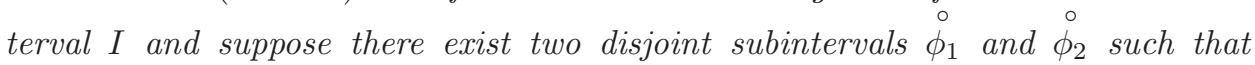


$\phi_{1} \cup \phi_{2}=I, f\left(\stackrel{\circ}{\phi_{1}}\right)=\stackrel{\circ}{\phi_{1}}$, and $f\left(\stackrel{\circ}{\phi}_{2}\right)={\stackrel{\circ}{\phi_{2}}}_{2}$. Then $S A F(f)=S A F\left(f_{\circ_{\phi_{1}}}\right)+S A F\left(f_{\phi_{2}}\right)$, where $f_{\stackrel{\phi}{i}_{i}}$ denotes the first return map to $\stackrel{\circ}{\phi}_{i}$.

In $[\underline{K S}$, Kenyon and Smillie prove the following theorem about the SAF invariant of an interval exchange transformation arising from a foliation of a translation surface.

Theorem 3.3. Let $S$ be a translation surface. Then $J_{x x}$ is the $S A F$ invariant of the vertical foliation of $S$, and $J_{y y}$ is the $S A F$ invariant of the horizontal foliation of $S$.

Furthermore, the following may also be found in $[\mathrm{A}$.

Theorem 3.4 (Arnoux). Consider an oriented foliation of a translation surface $S$. If a transverse interval I meets every leaf of the foliation, then the SAF invariant of $f_{I}$, the first return map to $I$, is independent of the interval $I$ chosen.

Finally, we state a lemma about specific types of 2- and 3-iets and their SAF invariants, and then we use this to obtain the two main lemmas which are used to show that any genus two surface which has Property X must be completely periodic.

Lemma 3.3. If $f$ is an interval exchange transformation on either two or three intervals and if $S A F(f)=0$, then $f$ is periodic.

Proof. See the Appendix.

Lemma 3.4. Let $S$ have genus 2 and fix a direction $v$ on $S$. Let $I$ be an interval transverse to the foliation in the direction $v$ which meets every leaf of the foliation and suppose that $S A F\left(f_{I}\right)=0$ where $f_{I}$ is the first return map to I. If there exists a cylinder of closed trajectories in this direction, $v$ must be a completely periodic direction on $S$.

Proof. First suppose $S \in \mathcal{H}(2)$. If we know that $S$ has a cylinder of closed trajectories, we choose an interval $I$ meeting every leaf of the foliation and such that the trajectories emitted from the endpoints hit the singularity before returning to $I$. We can choose a subinterval $I^{\prime \prime}$ of $I$ which passes through each trajectory of the cylinder and is bounded by saddle connections and assume that $I^{\prime}=I \backslash I^{\prime \prime}$ is also an interval. Then $I^{\prime}$ is the subinterval of $I$ which does not meet the cylinder but passes through every other trajectory and whose endpoints eventually hit the singularity before returning. By Theorem 3.2, $S A F\left(f_{I^{\prime \prime}}\right)=0$. Since $S A F\left(f_{I}\right)=0$, Lemma 3.2 implies $S A F\left(f_{I^{\prime}}\right)=0$ as well. But Corollary 3.1 implies $I^{\prime}$ has at most two subintervals associated to the first return map $f_{I^{\prime}}$. Since $S A F\left(f_{I^{\prime}}\right)=0$, Lemma 3.3 implies that $f_{I^{\prime}}$ is periodic. So the restriction of $f_{I}$ to each of the subintervals $I^{\prime \prime}$ and $I^{\prime}$ is periodic, and hence $f_{I}$ is periodic as well. Thus $S$ decomposes as a union of cylinders in this direction.

Now suppose $S \in \mathcal{H}(1,1)$. Choose $I$ and $I^{\prime \prime}$ as before. Corollary 3.1 implies $I$ is partitioned into at most five subintervals by $f_{I}$. Then since $f_{I^{\prime \prime}}$ is periodic, Theorem 3.2 yields $S A F\left(f_{I^{\prime \prime}}\right)=0$. Now let $I^{\prime}=I \backslash I^{\prime \prime}$. By the way we have chosen $I$ and $I^{\prime \prime}, I^{\prime}$ is partitioned into at most three subintervals. Now Lemma 3.2 implies $S A F\left(f_{I^{\prime}}\right)=0$. But $f_{I^{\prime}}$ is an iet on at most three intervals. Thus Lemma 3.3 implies that $f_{I^{\prime}}$ is completely periodic. Since $f_{I^{\prime \prime}}$ is also completely periodic, we have that $f_{I}$ is completely periodic and hence $S$ decomposes as a union of cylinders in the direction $v$ bounded by saddle connections. 


\section{Property X}

Given the definitions of $J_{v v}(S)$ and $J_{v w}(S)$ for arbitrary directions $v$ and $w$ on a translation surface $S$, we define a certain property of translation surfaces which we will use as an intermediate step in the proofs of our main results.

Definition 1. A direction $v$ on a translation surface $S$ is homological if there exists $\lambda \in H_{1}(S, \mathbb{Z})$ and $r \in \mathbb{R}$ such that $r v=p(\lambda)$. Note that $\lambda$ is not assumed to be simple.

Definition 2. A translation surface $S$ has Property $\mathbf{X}$ if for any homological direction $v$ on $S, J_{v v}(S)=0$.

In this section, we prove that for genus 2, Property $\mathrm{X}$ is equivalent to complete periodicity in Theorem 4.1 and Theorem 4.2 In Proposition 4.1 and Corollary 4.2 we determine a useful reformulation of Property $\mathrm{X}$ for surfaces in genus 2 . In order to do so, we assume that the translation surfaces are quadratic so that $p\left(H_{1}(S, \mathbb{Z})\right) \subset$ $\mathbb{Q}(\sqrt{d}) \times \mathbb{Q}(\sqrt{d})$. Notice that if this is the case, then if $\left(\begin{array}{l}1 \\ q\end{array}\right)$ is a vector representing a homological direction, $q \in \mathbb{Q}(\sqrt{d})$. Furthermore, the parameters $w_{i}, h_{i}, t_{i}$ of any cylinder decomposition of a surface in $\mathcal{H}(2)$ and the parameters $w_{i}, s_{i}, \tau_{i}$ of any cylinder decomposition of a surface in $\mathcal{H}(1,1)$ must lie in $\mathbb{Q}(\sqrt{d})$. At the end of the section, we show that in fact any genus two completely periodic translation surface (equivalently, any genus two surface having Property X) can be rescaled so that it is quadratic.

In the Appendix we show separately that any genus two, completely periodic surface $S$ having a one-cylinder decomposition in some direction can always be rescaled so that $p\left(H_{1}(S, \mathbb{Z})\right) \subset \mathbb{Q} \times \mathbb{Q}$. Thus for the rest of this section, our results will only involve two-cylinder decompositions of surfaces in $\mathcal{H}(2)$ as well as two- or three-cylinder decompositions of surfaces in $\mathcal{H}(1,1)$.

Theorem 4.1. If a genus two translation surface has Property $X$, then it is completely periodic.

Proof. We must show that if a genus two surface $S$ having Property $\mathrm{X}$ has a direction $v$ in which there is a cylinder of closed trajectories bounded by saddle connections, then $S$ decomposes as a union of cylinders in the direction $v$. In order to prove this, we will use the relationship between interval exchange transformations and foliations of surfaces described in Section 2.

Since $S$ has Property X, $J_{v v}(S)=0$. Applying an element of $S L(2, \mathbb{R})$, we may assume $v$ is the horizontal direction. Then Theorem $\left[2.2\right.$ implies that $J_{y y}(S)=0$. Furthermore, if $I$ is a vertical interval transverse to the foliation which meets every leaf, Theorem 3.3 implies that $S A F\left(f_{I}\right)=J_{y y}(S)=0$.

Since we are assuming there exists a cylinder of closed trajectories in the horizontal direction, and since $S A F\left(f_{I}\right)=0$ where $I$ is any transverse interval which meets every leaf of the foliation, Lemma [3.4 implies the surface decomposes as a union of cylinders of closed trajectories bounded by saddle connections. Thus $S$ itself is completely periodic. This completes the proof.

Theorem 4.2. Any genus 2 completely periodic translation surface has Property $X$. In particular, any Veech surface has Property $X$.

Proof. First suppose $S \in \mathcal{H}(2)$ is completely periodic. We may assume that the horizontal, vertical, and $\left(\begin{array}{l}1 \\ 1\end{array}\right)$ directions are completely periodic, and that $S$ has a 

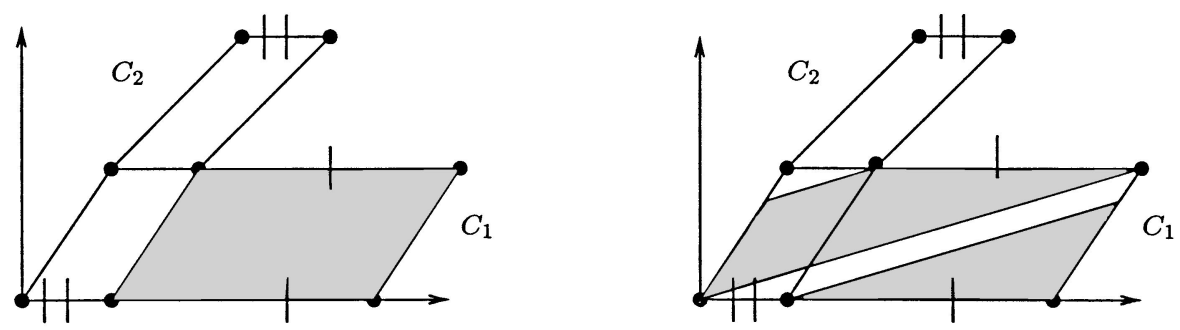

Figure 4. Cylinders for the vectors $\left(t_{1}, h_{1}\right)$ and $\left(t_{1}+1, h_{1}\right)$

two-cylinder decomposition in the horizontal direction. Let $w_{1}$ be the width of the widest cylinder. Then we can rescale the surface by dividing each coordinate of each point by $w_{1}$ without changing the fact that the three aforementioned directions are completely periodic. Thus we will assume $w_{1}=1$. We claim that there exist integers $k, n_{1}$ and $n_{2}, n_{1} \neq n_{2}$, such that the vectors $\left(t_{1}+t_{2}+n_{i} w_{2}, h_{1}+h_{2}\right)$, $\left(t_{1}, h_{1}\right),\left(t_{1}+1, h_{1}\right)$ and $\left(t_{1}+t_{2}+1+k w_{2}, h_{1}+h_{2}\right)$ all represent the core curves of cylinders of closed trajectories. (Recall that we are assuming $w_{1}=1$.) Assuming the claim is true, we will show how the claim implies that $S$ has Property $\mathrm{X}$ and then return to the proof of the claim.

Given the claim, the vectors $\left(t_{1}+t_{2}+n_{i} w_{2}, h_{1}+h_{2}\right),\left(t_{1}, h_{1}\right),\left(t_{1}+1, h_{1}\right)$ and $\left(t_{1}+\right.$ $\left.t_{2}+1+k w_{2}, h_{1}+h_{2}\right)$ all represent the core curves of cylinders on $S$. Since $S$ is completely periodic, Theorem 2.2 implies that $J$ must vanish in each of the directions $h_{1} / t_{1}, h_{1} /\left(1+t_{1}\right),\left(h_{1}+h_{2}\right) /\left(t_{1}+t_{2}+n_{i} w_{2}\right)$ and $h_{1}+h_{2} /\left(t_{1}+t_{2}+1+k w_{2}\right)$. We can apply Corollary 4.5, which appears later in this section, to conclude that each of these directions must lie in the field $F$ of directions in which $J$ vanishes. Thus, if $t_{1} \neq 0$, there exist $g_{0}, g_{1} \in F$ such that

$$
\begin{aligned}
& h_{1}=g_{0} t_{1}, \\
& h_{1}=g_{1}\left(1+t_{1}\right) .
\end{aligned}
$$

Together, (4.1) and (4.2) imply that if $t_{1} \neq 0$, then $t_{1}=g_{1} /\left(g_{0}-g_{1}\right)$, and hence $t_{1} \in F$. It follows that $h_{1} \in F$ as well. (If $t_{1}=0$, note that (4.2) implies $h_{1} \in F$.) Since the directions $\left(h_{1}+h_{2}\right) /\left(t_{1}+t_{2}+n_{i} w_{2}\right)$ and $h_{1}+h_{2} /\left(t_{1}+t_{2}+1+k w_{2}\right)$ lie in $F$ as well, there exist $f_{0}, f_{1}, f_{2} \in F$ such that

$$
\begin{aligned}
& h_{1}+h_{2}=f_{0}\left(t_{1}+t_{2}+n_{1} w_{2}\right), \\
& h_{1}+h_{2}=f_{1}\left(t_{1}+t_{2}+n_{2} w_{2}\right), \\
& h_{1}+h_{2}=f_{2}\left(t_{1}+t_{2}+1+k w_{2}\right) .
\end{aligned}
$$

Since $h_{1}, t_{1} \in F$, solving (4.3), (4.4) and (4.5) simultaneously implies that $h_{2}, t_{2}$ and $w_{2}$ are in $F$ as well. Since the classes of curves correpsonding to the vectors $\left(t_{i}, h_{i}\right)$ and $\left(w_{i}, 0\right)$ form a basis for $H_{1}(S, \mathbb{Z})$ and since each horizontal cylinder parameter $w_{i}, h_{i}, t_{i}$ lies in $F$, it follows that every homological direction must also lie in $F$ and so $J_{v v}=0$ for every homological direction $v$. Thus $S$ has Property X.

Now we return to the proof of the claim. Referring to shaded region in Figure 4 note that it is clear that the vector $\left(t_{1}, h_{1}\right)$ represents a cylinder of closed trajectories. Furthermore, by Dehn twisting each of the curves comprising this cylinder about the core curve for the horizontal cylinder $C_{1}$, we obtain another cylinder of closed trajectories for the vector $\left(t_{1}+1, h_{1}\right)$. 

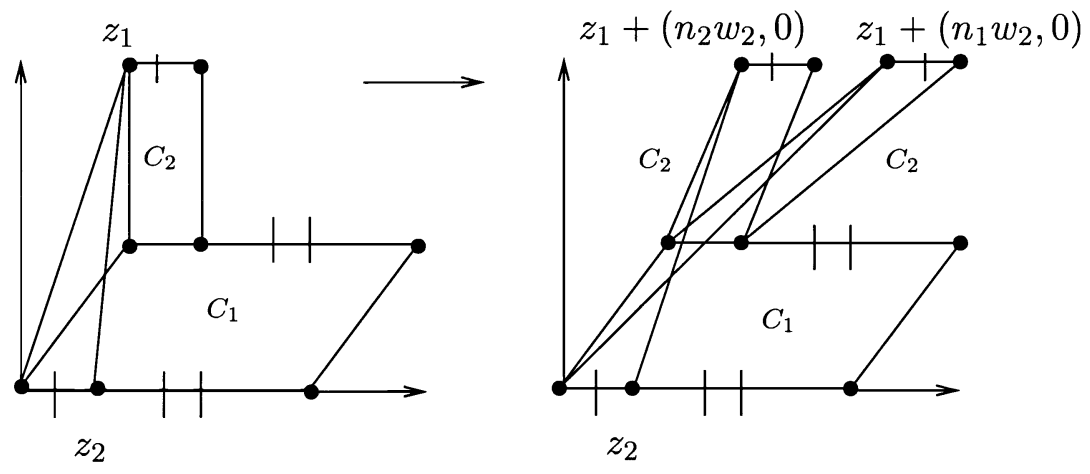

FiguRE 5. Saddle connections for the vectors $\left(t_{1}+t_{2}+n_{i} w_{2}, h_{1}+h_{2}\right)$

Although the vectors $\left(t_{1}+t_{2}, h_{1}+h_{2}\right)$ and $\left(t_{1}+t_{2}+w_{2}, h_{1}+h_{2}\right)$ do represent unions of two closed saddle connections, we require an entire cylinder of closed trajectories corresponding to each of these vectors. We will show that by adding $n_{1} w_{2}$ and $n_{2} w_{2}$, certain integer multiples of the width $w_{2}$, we may assume that the vectors $\left(t_{1}+t_{2}+n_{1} w_{2}, h_{1}+h_{2}\right)$ and $\left(t_{1}+t_{2}+n_{2} w_{2}, h_{1}+h_{2}\right)$ do represent cylinders of closed trajectories. In fact, we will find closed saddle connections representing each of these two vectors, and, referring to Figure 5 it is clear that there is a cylinder of closed trajectories for each of these vectors as well since a nonsingular closed geodesic determines an entire cylinder of closed, parallel, freely homotopic trajectories. In order to find a closed saddle connection whose holonomy is given by a vector of the form $\left(t_{1}+t_{2}+n_{1} w_{2}, h_{1}+h_{2}\right)$, we start by forming the vector $\left(t_{1}+t_{2}, h_{1}+h_{2}\right)$ in the plane which begins at the origin and connects the singularity to itself at the point $z_{1}=\left(t_{1}+t_{2}, h_{1}+h_{2}\right)$. We will show that there exists an integer $n_{1}$ such that the vector $\left(t_{1}+t_{2}+n_{1} w_{2}, h_{1}+h_{2}\right)$ with initial point at the origin and terminus at the point $z_{1}+n_{1} w_{2}$ passes from $C_{1}$ to $C_{2}$ between the singularities at $\left(t_{1}, h_{1}\right)$ and $\left(t_{1}+w_{2}, h_{1}\right)$. In other words, we seek an integer $n_{1}>0$ such that the line $y=\left(\left(h_{1}+h_{2}\right) /\left(t_{1}+t_{2}+n_{1} w_{2}\right)\right) x$ intersects the line $y=h_{1}$ between $x=t_{1}$ and $x=t_{1}+w_{2}$. These two lines intersect at the point $x=\left(\left(h_{1}\right) /\left(h_{2}+h_{1}\right)\right)\left(t_{1}+t_{2}+n_{1} w_{2}\right)$. Thus we wish to find $n_{1}>0$ such that

$$
t_{1}<\frac{h_{1}}{h_{1}+h_{2}}\left(t_{1}+t_{2}+n_{1} w_{2}\right)<t_{1}+w_{2} .
$$

But (4.6) is equivalent to

$$
\frac{h_{1}+h_{2}}{h_{1}}\left(\frac{t_{1}}{w_{2}}\right)-\frac{t_{1}+t_{2}}{w_{2}}<n_{1}<\frac{h_{1}+h_{2}}{h_{1}}\left(\frac{t_{1}}{w_{2}}\right)-\frac{t_{1}+t_{2}}{w_{2}}+\frac{h_{1}+h_{2}}{h_{1}} .
$$

Since $\left(h_{1}+h_{2}\right) / h_{1}>1$, we can always find an $n_{1}$ satisfying (4.7).

In order to find a second integer $n_{2}$, distinct from $n_{1}$, such that $\left(t_{1}+t_{2}+\right.$ $n_{2} w_{2}, h_{1}+h_{2}$ ) represents a cylinder, we use essentially the same argument above, except this time we find a closed saddle connection starting at the singularity $z_{2}=$ $\left(w_{2}, 0\right)$ and ending at the point $z_{1}+\left(n_{2} w_{2}, 0\right)=\left(t_{1}+t_{2}+n_{2} w_{2}, h_{1}+h_{2}\right)$. As before, we seek an integer $n_{2}$ such that the line $y=\left(\left(h_{1}+h_{2}\right) /\left(t_{1}+t_{2}+n_{2} w_{2}-w_{2}\right)\right)\left(x-w_{2}\right)$ intersects the line $y=h_{1}$ between $x=t_{1}$ and $x=t_{1}+w_{2}$. This means we seek an 


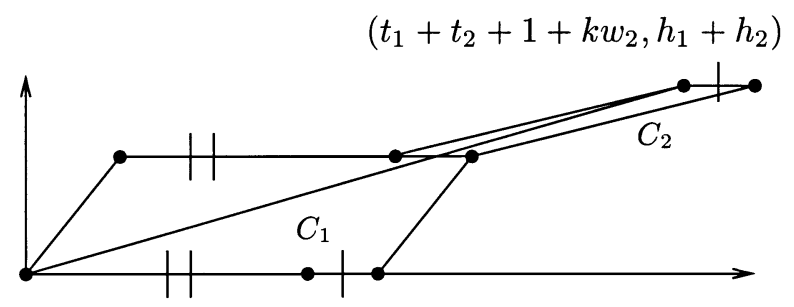

Figure 6 . A cylinder for the vector $\left(t_{1}+t_{2}+1+k w_{2}, h_{1}+h_{2}\right)$

integer $n_{2}$ satisfying

$$
t_{1}<\frac{h_{1}}{h_{1}+h_{2}}\left(t_{1}+t_{2}+\left(n_{2}-1\right) w_{2}\right)+w_{2}<t_{1}+w_{2} .
$$

But (4.8) is equivalent to

$$
\frac{h_{2}+h_{1}}{h_{1}}\left(\frac{t_{1}}{w_{2}}\right)-\frac{t_{1}+t_{2}}{w_{2}}+1-\frac{h_{2}+h_{1}}{h_{1}}<n_{2}<\frac{h_{2}+h_{1}}{h_{1}}\left(\frac{t_{1}}{w_{2}}\right)-\frac{t_{1}+t_{2}}{w_{2}}+1
$$

Since $\left(h_{2}+h_{1}\right) /\left(h_{1}\right)>1$, we can always find an $n_{2}$ satisfying (4.9).

Finally, we wish to find and integer $k$ such that the vector $\left(t_{1}+t_{2}+1+k w_{2}, h_{1}+h_{2}\right)$ represents a cylinder of closed trajectories. We use the same method here that we did to find $n_{1}$ and $n_{2}$. Referring to Figure 6, we wish to find an integer $k$ such that the line given by the equation $y=\left(h_{1}+h_{2}\right) /\left(t_{1}+t_{2}+1+k w_{2}\right)$ intersects the line $y=h_{1}$ between the points $x=t_{1}+1-w_{2}$ and $x=t_{1}+1$. This amounts to finding an integer $k$ satisfying

$$
t_{1}+1-w_{2}<\frac{h_{1}}{h_{1}+h_{2}}\left(t_{1}+t_{2}+1+k w_{2}\right)<t_{1}+1 .
$$

Note that (4.10) is equivalent to

$\frac{h_{1}+h_{2}}{h_{1}}\left(\frac{t_{1}+1}{w_{2}}\right)-\frac{1+t_{1}+t_{2}}{w_{2}}-\frac{h_{1}+h_{2}}{h_{1}}<k<\frac{h_{1}+h_{2}}{h_{1}}\left(\frac{t_{1}+1}{w_{2}}\right)-\frac{1+t_{1}+t_{2}}{w_{2}}$.

Again, since $\left(h_{1}+h_{2}\right) /\left(h_{1}\right)>1$, we can find an integer $k$ satisfying (4.11). This completes the proof of the claim.

Now suppose $S \in \mathcal{H}(1,1)$ has a three horizontal cylinder decomposition and that the vertical and $\left(\begin{array}{l}1 \\ 1\end{array}\right)$ directions are completely periodic as well. (The case when $S \in \mathcal{H}(1,1)$ has a horizontal two-cylinder decomposition is analogous and so we omit it.) The proof in this case mirrors the proof for the case when $S \in \mathcal{H}(2)$. Numbering the horizontal cylinders so that $w_{3}=w_{2}+w_{1}$, assume that $w_{1}$ is the width of the widest of the two cylinders $C_{1}$ and $C_{2}$. As before, rescale the surface so that $w_{1}=1$. We will show that there exist integers $n_{1} \neq n_{2}, m_{1} \neq m_{2}$ and $k$ so that the vectors $\left(\tau_{1}+n_{i}, s_{1}\right),\left(\tau_{2}+m_{i} w_{2}, s_{2}\right)$ and $\left(\tau_{1}+w_{2}+k, s_{1}\right)$ all represent cylinders of closed trajectories. Then each of the directions corresponding to these vectors must lie in $F$ and the argument used above to show that the parameters $w_{i}$, $t_{i}$, and $h_{i}$ lie in $F$ holds in this case as well, except that $t_{i}$ and $h_{i}$ are replaced with $\tau_{i}$ and $s_{i}$. As before, since the classes of curves corresponding to the vectors $\left(\tau_{i}, s_{i}\right)$ and $\left(w_{i}, 0\right), i=1$ or 2 , generate $H_{1}(S, \mathbb{Z})$, this implies that $p\left(H_{1}(S, \mathbb{Z})\right) \subset F \times F$ and so $S$ has Property X. 


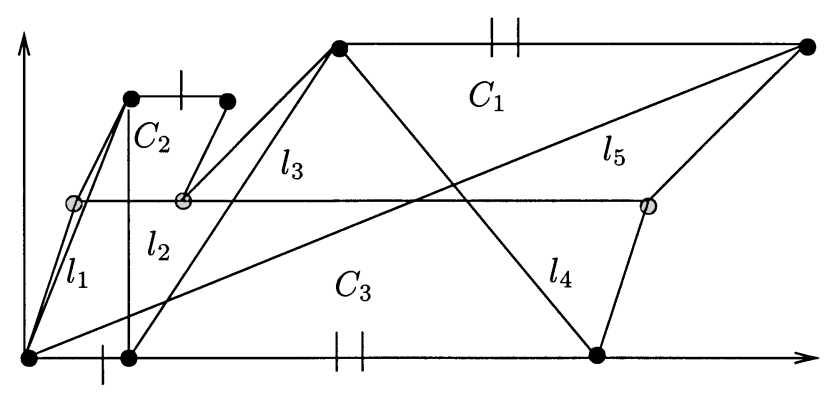

Figure 7. Closed saddle connections for a horizontal cylinder decomposition of $S \in \mathcal{H}(1,1)$

In order to show the existence of integers $m_{i}, n_{i}$, and $k$ such that each of the vectors $\left(\tau_{1}+n_{i}, s_{1}\right),\left(\tau_{2}+m_{i} w_{2}, s_{2}\right)$ and $\left(\tau_{1}+w_{2}+k, s_{1}\right)$ corresponds to a cylinder of closed trajectories, we use exactly the same argument as we used for the case $S \in \mathcal{H}(2)$ to find $n_{i}$ and $k$ such that the vectors $\left(t_{1}+t_{2}+n_{i} w_{2}, h_{1}+h_{2}\right)$ and $\left(t_{1}+t_{2}+1+k w_{2}, h_{1}+h_{2}\right)$ represented cylinders of closed trajectories. Referring to Figure7, the saddle connections $l_{1}, l_{2}, l_{3}, l_{4}$ and $l_{5}$ (each of which bounds a cylinder of closed trajectories) correspond to the vectors $\left(\tau_{2}+m_{i} w_{2}, s_{2}\right),\left(\tau_{1}+n_{i}, s_{1}\right)$ and $\left(\tau_{1}+k+w_{2}, s_{1}\right)$.

In the remainder of this section, we obtain a useful reformulation of Property $\mathrm{X}$ in genus 2, assuming such surfaces are quadratic, and we then prove that any genus 2 surface having Property X can be rescaled so that it is quadratic.

Proposition 4.1. Let $S$ be a genus two quadratic translation surface which cannot be rescaled so that $p\left(H_{1}(S, \mathbb{Z})\right) \subset \mathbb{Q} \times \mathbb{Q}$. Let $v$ and $w$ be some pair of completely periodic directions, so that $J_{v v}=J_{w w}=0$. We may write $J_{v w}(S)=c_{1}(1 \otimes 1)+$ $c_{2}(1 \otimes \sqrt{d})+c_{3}(\sqrt{d} \otimes 1)+c_{4}(\sqrt{d} \otimes \sqrt{d})$ where $c_{i} \in \mathbb{Q}$. If $S$ has Property $X$, then $c_{2}=c_{3}$ and $c_{1}=d c_{4}$. Conversely, if $S$ has Property $X$, then for any pair of completely periodic directions we have that $c_{2}=c_{3}$ and $c_{1}=d c_{4}$.

Proof. After applying an element of $S L(2, \mathbb{Q}(\sqrt{d}))$, we can assume that $v=x$ is the horizontal direction and $w=y$ the vertical. We will work out the case where $S \in \mathcal{H}(2)$ has a two-cylinder decompostion in the horizontal direction. However, note that Lemma 2.2 implies that the formula for $J(S)$ when $S \in \mathcal{H}(1,1)$ is the same as that for $S \in \mathcal{H}(2)$ with $h_{i}$ and $t_{i}$ replaced by $s_{i}$ and $\tau_{i}$, respectively. Since the proof of this proposition depends only on the formula for $J(S)$, the same argument will hold for a two- or three-cylinder decomposition of a surface $S \in \mathcal{H}(1,1)$.

Since we are assuming that $S$ has a cylinder decomposition in the horizontal direction, Lemma 2.2 implies

$$
J=2\left(\left(\begin{array}{c}
w_{1} \\
0
\end{array}\right) \wedge\left(\begin{array}{c}
t_{1} \\
h_{1}
\end{array}\right)+\left(\begin{array}{c}
w_{2} \\
0
\end{array}\right) \wedge\left(\begin{array}{c}
t_{2} \\
h_{2}
\end{array}\right)\right) .
$$

Thus, we see that

$$
J_{x x}=2\left(w_{1} \wedge t_{1}+w_{2} \wedge t_{2}\right)=0
$$

and

$$
J_{y y}=0 .
$$


Since $S$ is quadratic, we may write $w_{i}=w_{i}^{1}+w_{i}^{2} \sqrt{d}, t_{i}=t_{i}^{1}+t_{i}^{2} \sqrt{d}$, and $h_{i}=$ $h_{i}^{1}+h_{i}^{2} \sqrt{d}$ where $w_{i}^{j}, t_{i}^{j}, h_{i}^{j} \in \mathbb{Q}$ for $i, j \in\{1,2\}$. We find that

$$
\begin{aligned}
J_{x x} & =2\left(\left(w_{1}^{1}+w_{1}^{2} \sqrt{d}\right) \wedge\left(t_{1}^{1}+t_{1}^{2} \sqrt{d}\right)+\left(w_{2}^{1}+w_{2}^{2} \sqrt{d}\right) \wedge\left(t_{2}^{1}+t_{2}^{2} \sqrt{d}\right)\right) \\
& =2\left(w_{1}^{2} t_{1}^{1}-w_{1}^{1} t_{1}^{2}+w_{2}^{2} t_{2}^{1}-w_{2}^{1} t_{2}^{2}\right)(\sqrt{d} \wedge 1) .
\end{aligned}
$$

Thus, $J_{x x}=0$ if and only if

$$
w_{1}^{1} t_{1}^{2}+w_{2}^{1} t_{2}^{2}=w_{1}^{2} t_{1}^{1}+w_{2}^{2} t_{2}^{1} .
$$

Another calculation shows that

$$
\begin{aligned}
J_{x y} & =2\left(\left(w_{1}^{1} h_{1}^{1}+w_{2}^{1} h_{2}^{1}\right)(1 \otimes 1)+\left(w_{1}^{1} h_{1}^{2}+w_{2}^{1} h_{2}^{1}\right)(1 \otimes \sqrt{d})\right. \\
& \left.+\left(w_{1}^{2} h_{1}^{1}+w_{2}^{2} h_{2}^{1}\right)(\sqrt{d} \otimes 1)+\left(w_{1}^{2} h_{1}^{2}+w_{2}^{2} h_{2}^{2}\right)(\sqrt{d} \otimes \sqrt{d})\right) .
\end{aligned}
$$

Let $v=\left(\begin{array}{l}1 \\ q\end{array}\right), q=q_{1}+q_{2} \sqrt{d} \in \mathbb{Q}(\sqrt{d})$, be any homological direction on $S$, and suppose that $S$ has Property X. Recall that by definition, $J_{v v}(S)=0$ for any homological direction $v$ on $S$, where $J_{v v}(S)=J_{y y}(g S)$ and

$$
g=\left(\begin{array}{cc}
1 & 0 \\
-q & 1
\end{array}\right)
$$

Now,

$$
J(g S)=2\left(\left(\begin{array}{c}
w_{1} \\
-q w_{1}
\end{array}\right) \wedge\left(\begin{array}{c}
t_{1} \\
-q t_{1}+h_{1}
\end{array}\right)+\left(\begin{array}{c}
w_{2} \\
-q w_{2}
\end{array}\right) \wedge\left(\begin{array}{c}
t_{2} \\
-q t_{2}+h_{2}
\end{array}\right)\right) .
$$

Thus,

$$
\begin{aligned}
J_{v v}(S)=J_{y y}(g S) & =2\left(\left(-q w_{1}\right) \wedge\left(-q t_{1}+h_{1}\right)+\left(-q w_{2}\right) \wedge\left(-q t_{2}+h_{2}\right)\right) \\
& =2\left(\left(q w_{1} \wedge q t_{1}+q w_{2} \wedge q t_{2}\right)-\left(q w_{1} \wedge h_{1}+q w_{2} \wedge h_{2}\right)\right) .
\end{aligned}
$$

Note that since $w_{1} \wedge t_{1}+w_{2} \wedge t_{2}=0$, either there exist $r_{i} \in \mathbb{Q}$ such that

$$
\begin{aligned}
w_{2} & =r_{1} w_{1}+r_{2} t_{1}, \\
t_{2} & =r_{3} w_{1}+r_{4} t_{1},
\end{aligned}
$$

where $r_{1} r_{4}-r_{3} r_{2}=-1$, or there exist $p_{i} \in \mathbb{Q}$ such that

$$
w_{i}=p_{i} t_{i} .
$$

But the rational relations (4.15), (4.16) and (4.17) persist when $w_{i}$ and $t_{i}$ are replaced by $q w_{i}$ and $q t_{i}$, repsectively. Thus

$$
q w_{1} \wedge q t_{1}+q w_{2} \wedge q t_{2}=0,
$$

and so

$$
J_{v v}(S)=J_{y y}(g S)=-2\left(q w_{1} \wedge h_{1}+q w_{2} \wedge h_{2}\right) .
$$

A straightforward calculation yields

$$
-q w_{i}=-\left(q_{1} w_{i}^{1}+d q_{2} w_{i}^{2}\right)-\left(q_{1} w_{i}^{2}+q_{2} w_{i}^{1}\right) \sqrt{d} .
$$

Using (4.19), we have

$$
\begin{aligned}
\left(-q w_{i}\right) \wedge h_{i} & =\left(-\left(q_{1} w_{i}^{1}+d q_{2} w_{i}^{2}\right)-\left(q_{1} w_{i}^{2}+q_{2} w_{i}^{1}\right) \sqrt{d}\right) \wedge\left(h_{i}^{1}+h_{i}^{2} \sqrt{d}\right) \\
& =\left(-q_{1} w_{i}^{1}-d q_{2} w_{i}^{2}\right)\left(h_{i}^{2}\right)(1 \wedge \sqrt{d})+\left(-q_{1} w_{i}^{2}-q_{2} w_{i}^{1}\right)\left(h_{i}^{1}\right)(\sqrt{d} \wedge 1) \\
& =\left(q_{1}\left(w_{i}^{2} h_{i}^{1}-w_{i}^{1} h_{i}^{2}\right)+q_{2}\left(w_{i}^{1} h_{i}^{1}-d w_{i}^{2} h_{i}^{2}\right)\right)(1 \wedge \sqrt{d}) .
\end{aligned}
$$


Combining (4.20) and (4.18), we find that

$$
\begin{aligned}
J_{v v}(S)=J_{y y}(g S) & =-2\left(q_{1}\left(w_{1}^{2} h_{1}^{1}+w_{2}^{2} h_{2}^{1}-w_{1}^{1} h_{1}^{2}-w_{2}^{1} h_{2}^{1}\right)\right. \\
& \left.+q_{2}\left(w_{1}^{1} h_{1}^{1}+w_{2}^{1} h_{2}^{1}-d w_{1}^{2} h_{1}^{2}-d w_{2}^{2} h_{2}^{2}\right)\right)(1 \wedge \sqrt{d}) .
\end{aligned}
$$

Using the assumption about the homological directions of $S$, (4.21) and 4.13), we see that $S$ has Property $\mathrm{X}$ if and only if

$$
c_{1}=\left(w_{1}^{1} h_{1}^{1}+w_{2}^{1} h_{2}^{1}\right)=d\left(w_{1}^{2} h_{1}^{2}+w_{2}^{2} h_{2}^{2}\right)=d c_{4}
$$

and

$$
c_{2}=\left(w_{1}^{1} h_{1}^{2}+w_{2}^{1} h_{2}^{2}\right)=\left(w_{1}^{2} h_{1}^{1}+w_{2}^{2} h_{2}^{1}\right)=c_{3} .
$$

The following corollary is a direct result of the previous proposition.

Corollary 4.2. Let $S \subset \mathcal{H}(2)$ be a quadratic translation surface which cannot be rescaled so that $p\left(H_{1}(S, \mathbb{Z})\right) \subset \mathbb{Q} \times \mathbb{Q}$. Assume that $S$ has a two-cylinder decomposition in the horizontal direction. Let $w_{1}, w_{2}, h_{1}, h_{2}, t_{1}, t_{2}$ be the widths, heights and twists of these two cylinders. Then $J_{x x}=0$ if and only if

$$
\bar{w}_{1} t_{1}+\bar{w}_{2} t_{2}=w_{1} \bar{t}_{1}+w_{2} \bar{t}_{2} \text {. }
$$

Furthermore, the equations $c_{1}=d c_{4}$ and $c_{2}=c_{3}$ defining Property $X$ are equivalent to

$$
\begin{aligned}
w_{1} \bar{h}_{1} & =-w_{2} \bar{h}_{2}, \\
w_{1} h_{1}+w_{2} h_{2} & =2\left(c_{1}+c_{2} \sqrt{d}\right) .
\end{aligned}
$$

If $S \in \mathcal{H}(1,1)$ has either a two- or three-cylinder decomposition in the horizontal direction, then the same equations hold with $h_{i}$ replaced by $s_{i}=h_{i}+h_{3}$ and $t_{i}$ replaced by $\tau_{i}=t_{i}+t_{3}$ for $i=1,2$ and where the cylinders are numbered so that $w_{3}=w_{2}+w_{1}$.

Proof. We will prove the lemma in the case where $S \in \mathcal{H}(2)$ has a two-cylinder decomposition. The proof is the same if $S \in \mathcal{H}(1,1)$ with $h_{i}$ replaced by $s_{i}$ and $t_{i}$ replaced by $\tau_{i}$.

In the proof of the previous lemma, we saw that $J_{x x}=0$ if and only if

$$
w_{1}^{1} t_{1}^{2}+w_{2}^{1} t_{2}^{2}=w_{1}^{2} t_{1}^{1}+w_{2}^{2} t_{2}^{1} .
$$

Using the fact that $w_{i}=w_{i}^{1}+w_{i}^{2} \sqrt{d}$ and the similar definition of $t_{i}$, a short computation shows that (4.22) and (4.25) are equivalent. Thus $J_{x x}=0$ if and only if (4.22) holds. if

The proof of the previous lemma also showed that $S$ had Property X if and only

$$
c_{1}=\left(w_{1}^{1} h_{1}^{1}+w_{2}^{1} h_{2}^{1}\right)=d\left(w_{1}^{2} h_{1}^{2}+w_{2}^{2} h_{2}^{2}\right)=d c_{4}
$$

and

$$
c_{2}=\left(w_{1}^{1} h_{1}^{2}+w_{2}^{1} h_{2}^{2}\right)=\left(w_{1}^{2} h_{1}^{1}+w_{2}^{2} h_{2}^{1}\right)=c_{3} .
$$

Making the same substitutions into (4.23) and (4.24) as we did above for $w_{i}, h_{i}, t_{i}$ and their algebraic conjugates and then simplifying yields 4.26) and 4.27).

Finally, we prove that any genus 2 completely periodic surface is quadratic. 
Lemma 4.3. Suppose that the vertical, horizontal and $\left(\begin{array}{l}1 \\ 1\end{array}\right)$ directions on a genus two translation surface $S$ are completely periodic. If $S \in \mathcal{H}(2)$ decomposes into two cylinders in the horizontal direction, then $J_{x y}(S)$ is symmetric, and we can write

$$
J_{x y}(S)=w_{1} \otimes h_{1}+h_{1} \otimes w_{1}+w_{2} \otimes h_{2}+h_{2} \otimes w_{2} .
$$

If $S \in \mathcal{H}(1,1)$ decomposes into two or three horizontal directions, then $J_{x y}(S)$ is also symmetric and the corresponding equation

$$
J_{x y}(S)=2\left(h_{1} \otimes w_{1}+h_{2} \otimes w_{2}\right)
$$

holds.

Proof. We will prove the theorem when $S \in \mathcal{H}(2)$ has a two-cylinder decomposition in the horizontal direction. If $S \in \mathcal{H}(1,1)$ has a two- or three-cylinder decomposition, then the same proof holds with $h_{i}$ and $t_{i}$ replaced by $s_{i}$ and $\tau_{i}$ for $i=1,2$, respectively.

Since the horizontal direction on $S$ is completely periodic, Lemma 2.2 implies

$$
J(S)=2\left(\left(\begin{array}{c}
w_{1} \\
0
\end{array}\right) \wedge\left(\begin{array}{c}
t_{1} \\
h_{1}
\end{array}\right)+\left(\begin{array}{c}
w_{2} \\
0
\end{array}\right) \wedge\left(\begin{array}{c}
t_{2} \\
h_{2}
\end{array}\right)\right)
$$

and Corollary 2.3 implies

$$
J_{x y}(S)=2\left(w_{1} \otimes h_{1}+w_{2} \otimes h_{2}\right) .
$$

Since the direction $\left(\begin{array}{l}1 \\ 1\end{array}\right)$ on $S$ is completely periodic, Theorem 2.2 implies that $J_{y y}(g S)=0$ where

$$
g=\left(\begin{array}{cc}
1 & 0 \\
-1 & 1
\end{array}\right)
$$

i.e., $g\left(\begin{array}{l}1 \\ 1\end{array}\right)=\left(\begin{array}{l}1 \\ 0\end{array}\right)$. Note that

$$
J(g S)=2\left(\left(\begin{array}{c}
w_{1} \\
-w_{1}
\end{array}\right) \wedge\left(\begin{array}{c}
t_{1} \\
h_{1}-t_{1}
\end{array}\right)+\left(\begin{array}{c}
w_{2} \\
-w_{2}
\end{array}\right) \wedge\left(\begin{array}{c}
t_{2} \\
h_{2}-t_{2}
\end{array}\right)\right)
$$

and thus

$$
J_{y y}(g S)=2\left(-\left(w_{1} \wedge h_{1}+w_{2} \wedge h_{2}\right)+\left(w_{1} \wedge t_{1}+w_{2} \wedge t_{2}\right)\right)=0 .
$$

Since the vertical direction on $S$ is completely periodic as well, $w_{1} \wedge t_{1}+w_{2} \wedge t_{2}=0$. Thus

Equivalently,

$$
w_{1} \wedge h_{1}+w_{2} \wedge h_{2}=0 .
$$

$$
w_{1} \otimes h_{1}-h_{1} \otimes w_{1}+w_{2} \otimes h_{2}-h_{2} \otimes w_{2}=0 .
$$

Now, using (4.31), 4.30) can be rewritten as

$$
J_{x y}(S)=2\left(h_{1} \otimes w_{1}+h_{2} \otimes w_{2}\right)=w_{1} \otimes h_{1}+w_{2} \otimes h_{2}+h_{1} \otimes w_{1}+h_{2} \otimes w_{2} .
$$

This completes the proof.

Lemma 4.4. Let $S$ be a genus two translation surface such that the horizontal and vertical directions, along with the direction $\left(\begin{array}{l}1 \\ 1\end{array}\right)$, are completely periodic. Let $v=\left(\begin{array}{l}1 \\ q\end{array}\right)$ be any direction such that $J_{v v}(S)=0$. Then

$$
(1 \otimes q-q \otimes 1) J_{x y}(S)=0 .
$$


Proof. As in the proof of Lemma 4.3 we will assume $S \in \mathcal{H}(2)$ has a two-cylinder decomposition in the horizontal direction. If $S \in \mathcal{H}(1,1)$, the proof can be modified in the same way as the proof of Lemma 4.3. Since the horizontal direction on $S$ is completely periodic, Lemma 2.2 implies

$$
J(S)=2\left(\left(\begin{array}{c}
w_{1} \\
0
\end{array}\right) \wedge\left(\begin{array}{c}
t_{1} \\
h_{1}
\end{array}\right)+\left(\begin{array}{c}
w_{2} \\
0
\end{array}\right) \wedge\left(\begin{array}{c}
t_{2} \\
h_{2}
\end{array}\right)\right) .
$$

Now suppose that $\left(\begin{array}{l}1 \\ q\end{array}\right)$ is a completely periodic direction on $S$. Theorem 2.2 implies $J_{y y}(g S)=0$ where

$$
g=\left(\begin{array}{cc}
1 & 0 \\
-q & 1
\end{array}\right)
$$

so that $g\left(\begin{array}{l}1 \\ q\end{array}\right)=\left(\begin{array}{l}1 \\ 0\end{array}\right)$. Note that

$$
J(g S)=2\left(\left(\begin{array}{c}
w_{1} \\
-q w_{1}
\end{array}\right) \wedge\left(\begin{array}{c}
t_{1} \\
h_{1}-q t_{1}
\end{array}\right)+\left(\begin{array}{c}
w_{2} \\
-q w_{2}
\end{array}\right) \wedge\left(\begin{array}{c}
t_{2} \\
h_{2}-q t_{2}
\end{array}\right)\right)
$$

and thus

$$
J_{y y}(g S)=-2\left(q w_{1} \wedge h_{1}+q w_{2} \wedge h_{2}\right)+2\left(q w_{1} \wedge q t_{1}+q w_{2} \wedge q t_{2}\right)=0 .
$$

Since $w_{1} \wedge t_{1}+w_{2} \wedge t_{2}=0$, the proof of Proposition 4.1 implies

$$
q w_{1} \wedge q t_{1}+q w_{2} \wedge q t_{2}=0 .
$$

Then (4.33) implies

$$
q w_{1} \wedge h_{1}+q w_{2} \wedge h_{2}=0
$$

By definition, this yields

$$
q w_{1} \otimes h_{1}+q w_{2} \otimes h_{2}=h_{1} \otimes q w_{1}+h_{2} \otimes q w_{2} .
$$

Then Lemma 4.3 and (4.34) imply

$$
\begin{aligned}
(1 \otimes q-q \otimes 1) J_{x y}(S) & =2(1 \otimes q)\left(h_{1} \otimes w_{1}+h_{2} \otimes w_{2}\right) \\
& -2(q \otimes 1)\left(w_{1} \otimes h_{1}+w_{2} \otimes h_{2}\right) \\
& =0 .
\end{aligned}
$$

Corollary 4.5. With hypotheses as in Lemma 4.4, the set of numbers $F=\{q$ : $\left.(1 \otimes q-q \otimes 1) J_{x y}(S)=0\right\}$ is a field which contains $\mathbb{Q}$.

Proof. First, we will show that $\mathbb{Q} \subset F$. Let $q \in \mathbb{Q}$. Then $1 \otimes q=q \otimes 1$ and so $(1 \otimes q-q \otimes 1) J_{x y}(S)=0$. Hence $\mathbb{Q} \subset F$. Now suppose that $q_{1}, q_{2} \in F$. We will show that $q_{1}-q_{2}, q_{1} q_{2}$ and $q_{1}^{-1}$ are in $F$. If $q_{1}, q_{2} \in F$, then $\left(1 \otimes q_{i}-q_{i} \otimes 1\right) J_{x y}(S)=0$ for $i=1,2$ and thus

$$
\begin{aligned}
\left(1 \otimes\left(q_{1}-q_{2}\right)-\left(q_{1}-q_{2}\right) \otimes 1\right) J_{x y}(s) & =\left(1 \otimes q_{1}-q_{1} \otimes 1\right) J_{x y}(S) \\
& -\left(1 \otimes q_{2}-q_{2} \otimes 1\right) J_{x y}(S) \\
& =0 .
\end{aligned}
$$


So $q_{1}-q_{2} \in F$. Also, note that

$$
\begin{aligned}
\left(1 \otimes q_{1} q_{2}\right) J_{x y}(S) & =\left(1 \otimes q_{1}\right)\left(\left(1 \otimes q_{2}\right) J_{x y}(S)\right) \\
& =\left(1 \otimes q_{1}\right)\left(\left(q_{2} \otimes 1\right) J_{x y}(S)\right) \\
& =\left(q_{2} \otimes 1\right)\left(\left(1 \otimes q_{1}\right) J_{x y}(S)\right) \\
& =\left(q_{2} \otimes 1\right)\left(\left(q_{1} \otimes 1\right) J_{x y}(S)\right) \\
& =\left(q_{1} q_{2} \otimes 1\right) J_{x y}(S) .
\end{aligned}
$$

Thus $\left(1 \otimes q_{1} q_{2}-q_{1} q_{2} \otimes 1\right) J_{x y}(S)=0$ and so $q_{1} q_{2} \in F$ as well. Finally, to show that $q_{1}^{-1} \in F$, note that since $\left(1 \otimes q_{1}-q_{1} \otimes 1\right) J_{x y}(S)=0$ and $J_{x y}(S)=2\left(w_{1} \otimes h_{1}+w_{2} \otimes h_{2}\right)$, the proof of Lemma 4.4 shows that

$$
q w_{1} \wedge h_{1}+q w_{2} \wedge h_{2}=0
$$

and so

$$
w_{1} \wedge q_{1}^{-1} h_{1}+w_{2} \wedge q_{1}^{-1} h_{2}=0 .
$$

Now (4.351) can be rewritten as

$$
w_{1} \otimes q_{1}^{-1} h_{1}+w_{2} \otimes q_{1}^{-1} h_{2}-q_{1}^{-1} h_{1} \otimes w_{1}-q_{1}^{-1} h_{2} \otimes w_{2}=0 .
$$

Using (4.36), we find that

$$
\begin{aligned}
\left(1 \otimes q_{1}^{-1}-q_{1}^{-1} \otimes 1\right) J_{x y}(S) & =\left(1 \otimes q_{1}^{-1}\right)\left(2\left(w_{1} \otimes h_{1}+w_{2} \otimes h_{2}\right)\right) \\
& -\left(q_{1}^{-1} \otimes 1\right)\left(2\left(h_{1} \otimes w_{1}+h_{2} \otimes w_{2}\right)\right) \\
& =2\left(w_{1} \otimes q_{1}^{-1} h_{1}+w_{2} \otimes q_{1}^{-1} h_{2}\right) \\
& -2\left(q_{1}^{-1} h_{1} \otimes w_{1}-q_{1}^{-1} h_{2} \otimes w_{2}\right) \\
& =0 .
\end{aligned}
$$

This completes the proof.

Theorem 4.3. Any genus two completely periodic translation surface is quadratic.

Proof. Note that we may assume the horizontal, vertical, and $\left(\begin{array}{l}1 \\ 1\end{array}\right)$ directions on $S$ are periodic. In $\mathrm{Ma}$, Masur proved that the set of directions in which there is a cylinder of closed trajectories is dense. In particular, there must be a triple of transverse periodic directions, $u, v, w$. Then there exists a $g \in S L(2, \mathbb{R})$ such that the horizontal, vertical and $\left(\begin{array}{l}1 \\ 1\end{array}\right)$ directions are periodic on the (completely periodic) surface $g S$. We will prove the theorem in the case where $S \in \mathcal{H}(2)$ has a horizontal two-cylinder decomposition with parameters $w_{i}, h_{i}$ and $t_{i}$. The proof in the case where $S \in \mathcal{H}(1,1)$ has either a two- or three-cylinder horizontal decomposition is the same, with $h_{i}$ and $t_{i}$ replaced by $s_{i}$ and $\tau_{i}$, respectively. Furthermore, we can rescale the surface by dividing each coordinate of each point by the quantity $w_{1}$, which will not change the fact that the horizontal, vertical, and $\left(\begin{array}{l}1 \\ 1\end{array}\right)$ directions are completely periodic. Thus we will assume $w_{1}=1$ and let $w_{2}=w$. The proof of Lemma 4.3 implies that

$$
1 \wedge h_{1}+w \wedge h_{2}=0
$$

and if $\left(\begin{array}{l}1 \\ q\end{array}\right)$ is any direction along which $J$ vanishes, then the proof of Lemma 4.4 yields

$$
q \wedge h_{1}+q w \wedge h_{2}=0 .
$$


Our strategy will be to use (4.37) and (4.38) to obtain information about the linear relations among the numbers $1, w, h_{1}$ and $h_{2}$. Now the proof of Theorem 4.2 implies that $w, h_{1}, h_{2}$ must lie in the field $F$ of directions along which $J$ vanishes. Combining this fact, along with the rational relations resulting from (4.37) and (4.38), we will show that $w$ must be quadratic (if it is not rational), and hence so must $h_{1}$ and $h_{2}$ be.

First suppose that $w \in \mathbb{Q}$. Then (4.37) implies that $1 \wedge\left(h_{1}+w h_{2}\right)=0$ and so there exists $r \in \mathbb{Q}$ such that $h_{1}+w h_{2}=r$. Using (4.38), we see that $q \in \mathbb{Q}$, so $F=\mathbb{Q}$. But then all parameters $w, t_{i}$ and $h_{i}$ are rational as well.

Now suppose that $w$ is not in $\mathbb{Q}$. Then 4.37) implies that either there exist rational numbers $r_{1}, r_{2}, r_{3}$ and $r_{4}$ such that

$$
\begin{aligned}
w & =r_{1}+r_{2} h_{1}, \\
h_{2} & =r_{3}+r_{4} h_{1}
\end{aligned}
$$

where $r_{1} r_{4}-r_{2} r_{3}=-1$ or there exist $p_{i} \in \mathbb{Q}, i=1,2$, such that

$$
\begin{aligned}
h_{1} & =p_{1}, \\
w & =p_{2} h_{2} .
\end{aligned}
$$

In either case, note that $h_{i} \in \mathbb{Q}[1, w]$. Since (4.38) holds as well, and since we may take $q=w$, either we have that there exist rational numbers $r_{i}^{\prime}$ such that

$$
\begin{gathered}
w^{2}=r_{1}^{\prime} w+r_{2}^{\prime} h_{1}, \\
h_{2}=r_{3}^{\prime} w+r_{4}^{\prime} h_{1}
\end{gathered}
$$

or that there exist rational numbers $p_{i}^{\prime}$ such that

$$
\begin{aligned}
w & =p_{1}^{\prime} h_{1}, \\
w^{2} & =p_{2}^{\prime} h_{2} .
\end{aligned}
$$

If (4.43) holds, then we may substitute the appropriate expression for $h_{1}$ from either (4.39) or (4.41) to conclude that either

$$
w^{2}=\left(r_{1}^{\prime}+\frac{r_{2}^{\prime}}{r_{2}}\right) w-\frac{r_{2}^{\prime} r_{1}}{r_{2}}
$$

or

$$
w^{2}=r_{1}^{\prime} w+p_{1} r_{2}^{\prime} .
$$

In either case, $w \in \mathbb{Q}(\sqrt{d})$ and thus $h_{i}$ are as well. In the case that (4.46) holds, similar arguments show that $w$ and $h_{i}$ are quadratic.

Finally, since the vertical direction on $S$ is completely periodic, the proof of Lemma 4.4 implies that

$$
1 \wedge t_{1}+w \wedge t_{2}=0
$$

Then an argument similar to the above shows $t_{i} \in \mathbb{Q}[1, w]$ and so the twists are quadratic as well. Since the vectors $\left(\begin{array}{c}t_{i} \\ h_{i}\end{array}\right)$ and $\left(\begin{array}{c}w_{i} \\ 0\end{array}\right)$ generate $H_{1}(S, \mathbb{Z})$, we have that $p\left(H_{1}(S, \mathbb{Z})\right) \subset \mathbb{Q}(\sqrt{d}) \times \mathbb{Q}(\sqrt{d})$, and so $S$ must be quadratic. 


\section{The Classification of Veech surfaces in $\mathcal{H}(2)$}

Here we prove several theorems involving surfaces which lie in the stratum $\mathcal{H}(2)$, in particular a classification of Veech surfaces. In order to obtain this classification, we need the following lemma, which we will also use to classify completely periodic surfaces in the stratum $\mathcal{H}(1,1)$ in the following section.

Let $\mathcal{O}_{d}^{+}$denote the positive algebraic integers in $\mathbb{Q}(\sqrt{d})$.

Lemma 5.1. For fixed integers $c_{1}$ and $c_{2}$, such that $c_{1}$ is divisible by $d$, the equations

$$
\begin{aligned}
w_{1} \bar{h}_{1} & =-w_{2} \bar{h}_{2}, \\
\bar{w}_{1} t_{1}+\bar{w}_{2} t_{2} & =w_{1} \bar{t}_{1}+w_{2} \bar{t}_{2}, \\
w_{1} h_{1}+w_{2} h_{2} & =2\left(c_{1}+c_{2} \sqrt{d}\right)
\end{aligned}
$$

where $0 \leq t_{i} \leq w_{i}$ have finitely many solutions in $\mathcal{O}_{d}^{+}$, the positive algebraic integers in $\mathbb{Q}(\sqrt{d})$, up to the action by the group of units. Let us denote this finite number by $H\left(c_{1}, c_{2}\right)$.

Proof. See the Appendix.

We will also make use of the following two lemmas in the proof of the classification of Veech surfaces in $\mathcal{H}(2)$.

Lemma 5.2. Let $D(t)=\operatorname{diag}\left(t, t, t^{-1}, t^{-1}, t, t\right)$. Let $\Delta$ be a finite set in $\mathbb{R}^{6}$, and let $\mathcal{M}_{1}(\Delta)$ denote the set of completely periodic sufaces in $\mathcal{H}(2)$ such that the cylinder decomposition along any periodic direction $v$ satisfies $\left(w_{1}, w_{2}, h_{1}, h_{2}, t_{1}, t_{2}\right) \in$ $D(\mathbb{R}) \Delta$. Then, provided $\mathcal{M}_{1}(\Delta)$ is nonempty, $\mathcal{M}_{1}(\Delta)$ is a closed invariant subset of $\mathcal{H}(2)$.

Proof. First, we will show that the set $\mathcal{M}_{1}(\Delta)$ is invariant under the action of $S L(2, \mathbb{R})$. Let $S \in \mathcal{M}_{1}(\Delta)$ and $g \in S L(2, \mathbb{R})$. Since $S \in \mathcal{M}_{1}(\Delta)$, by definition, $S$ is completely periodic and the width, height, and twist parameters for $S$ in any completely periodic direction $v$ satisfy $\left(w_{1}, w_{2}, h_{1}, h_{2}, t_{1}, t_{2}\right) \in D(\mathbb{R}) \Delta$. Note that $g S$ is also completely periodic since the application of any element of $S L(2, \mathbb{R})$ preserves a cylinder decomposition. Thus, the completely periodic directions of $g S$ are precisely the directions $g v$ where $v$ is a completely periodic direction of $S$. Now, since any element of $S L(2, \mathbb{R})$ is area preserving, if a matrix $g$ multiplies the width $w_{i}$ of a cylinder by $t$, then it must multiply the height $h_{i}$ of the cylinder by $t^{-1}$. If the twists were measured in the completely periodic direction $v^{\prime}$ on $S$, then if we measure the twists on $g S$ in the direction $g v^{\prime}$, each twist parameter $t_{i}$ will also be multiplied by the factor $t$. Thus $\mathcal{M}_{1}(\Delta)$ is invariant under the action of $S L(2, \mathbb{R})$.

Now we must show that $\mathcal{M}_{1}(\Delta)$ is a closed subset of $\mathcal{H}(2)$. Suppose that we have a sequence of surfaces $\left\{S_{i}\right\} \subset \mathcal{M}_{1}(\Delta)$ which converges to a surface $S$. We must show that $S \in \mathcal{M}_{1}(\Delta)$. First, we will prove that $S$ is completely periodic, that is, if $v$ is any direction in which $S$ has a cylinder of closed trajectories with core curve $\gamma$, then $S$ decomposes as a union of cylinders in the direction $v$. Furthermore, we need to show that if $w_{S}^{i}, h_{S}^{i}, t_{S}^{i}$ for $i=1,2$ are the width, height and twist paramters for $S$ in the direction $v$, then they form a vector lying in $D(\mathbb{R}) \Delta$. For large enough $i$, on each surface $S_{i}$, there is a closed cylinder with core curve $\gamma_{i}$ such that $l_{S_{i}}\left(\gamma_{i}\right) \rightarrow l_{S}(\gamma)=w_{S}^{1}$. Define $w_{i}^{1}=l_{S_{i}}\left(\gamma_{i}\right)$. Since each $S_{i}$ is completely periodic, it decomposes into a union of two cylinders in a direction $v_{i}$ close to $v$. 
Thus, if $w_{i}^{1}$ is the width of one of these cylinders, then $w_{i}^{2}$ is the width of the second, and $h_{i}^{1}, t_{i}^{1}, h_{i}^{2}$, and $t_{i}^{2}$ are the corresponding height and twist parameters. Now recall that $\Delta$ is a finite set. Thus, upon passing to a subsequence, we may assume that $w_{i}^{j}=m_{i} w^{j}, h_{i}^{j}=m_{i}^{-1} h^{j}$, and $t_{i}^{j}=m_{i} t^{j}$ for $j=1,2$, where $\left(w^{1}, w^{2}, h^{1}, h^{2}, t^{1}, t^{2}\right)$ is a single vector in $\Delta$. Since $w_{i}^{1}=m_{i} w^{1} \rightarrow w_{S}^{1}, m_{i} \rightarrow m$ and $m \neq 0$. So $w_{i}^{j} \rightarrow m w^{j}, h_{i}^{j} \rightarrow m^{-1} h^{j}$, and $t_{i}^{j} \rightarrow m t^{j}$. In particular, since $m \neq 0, m h^{j} \neq 0$ for $j=1,2$. Using this convergence, the fact that $m h^{j} \neq 0$, and the fact that a cylinder decomposition on each $S_{i}$ is stable under small perturbations, we see that $S$ has a cylinder decomposition in the direction $v$ with width, height and twist parameters $m w^{j}, m^{-1} h^{j}$ and $m t^{j}$ for $j=1,2$. Thus, $\mathcal{M}_{1}(\Delta)$ is closed.

Lemma 5.3. If $S \in \mathcal{H}(2)$ has Property $X$, then its $S L(2, \mathbb{R})$ orbit is closed.

Proof. This will follow mainly from Lemma 5.2 First, we show that the $S L(2, \mathbb{R})$ orbit of $S$ is contained in $\mathcal{M}_{1}(\Delta)$ for some finite set $\Delta$ of $\mathbb{R}^{6}$. Since $S$ has Property X, Theorem 4.1 implies that $S$ is completely periodic. Since an element of $S L(2, \mathbb{R})$ preserves a cylinder decomposition, $g S$ is also completely periodic for any $g \in S L(2, \mathbb{R})$. Again, since $S$ has Property X, Corollary 4.2 implies that if $v$ is any completely periodic direction on $S$ such that the twists are measured along another completely periodic direction $v^{\prime}$, and $w_{i}, h_{i}, t_{i}$ are the width, height and twist parameters in the direction $v$, then (5.1), (5.2) and (5.3) are satisfied. Now Lemma 5.1 implies these equations have only finitely many solutions in $\mathcal{O}_{d}{ }^{+}$, up to the action by the group of units. Let $\Delta$ of Lemma 5.2 be this set of finitely many solutions. It follows that the vector $\left(w_{1}, w_{2}, h_{1}, h_{2}, t_{1}, t_{2}\right)$ formed by the width, height, and twist parameters of any cylinder decomposition of any surface in the $S L(2, \mathbb{R})$ orbit of $S$ must lie in the set $D(\mathbb{R}) \Delta$. Thus the $S L(2, \mathbb{R})$ orbit of $S$ lies in the set $\mathcal{M}_{1}(\Delta)$. But Lemma 5.2 implies that $\mathcal{M}_{1}(\Delta)$ is a closed set. In fact, the proof of Lemma 5.2 shows that each $S L(2, \mathbb{R})$ orbit of a surface in $\mathcal{M}_{1}(\Delta)$ is closed since two surfaces which have cylinder decompositions in some direction whose parameters are given by the vectors $\left(w_{1}, w_{2}, t_{1}, t_{2}, h_{1}, h_{2}\right)$ and $\left(m w_{1}, m w_{2}, m t_{1}, m t_{2}, m^{-1} h_{1}, m^{-1} h_{2}\right)$ are related by an element of $S L(2, \mathbb{R})$. This completes the proof.

Given these two lemmas, we have the following theorem.

Theorem 5.1. If a surface in $\mathcal{H}(2)$ has Property $X$, then it is Veech.

Proof. In order to show that $S$ is Veech, it suffices to show that the $S L(2, \mathbb{R})$ orbit of $S$ is closed. See $\mathrm{V} 2$ for the sketch of a proof of this fact by John Smillier. This is an immediate consequence of Lemma 5.3 .

Finally, we state our classification theorem.

Theorem 5.2. Let $S$ be a Veech surface in $\mathcal{H}(2)$ which cannot be rescaled so that $p\left(H_{1}(S, \mathbb{Z})\right) \subset \mathbb{Q} \times \mathbb{Q}$. Let $v$ be a completely periodic direction for $S$. Suppose the cylinder decomposition in the direction $v$ has two cylinders. Let $w_{1}, w_{2}, h_{1}, h_{2}, t_{1}, t_{2}$ be the widths, heights and twists of these two cylinders. The twists are measured along some direction $v^{\prime}$ which we assume is also completely periodic. After rescaling the surface, we may assume that these variables are algebraic integers in $\mathbb{Q}(\sqrt{d})$ where $d$ is some square-free, positive integer. Then the following equations are 
satisfied:

$$
\begin{aligned}
w_{1} \bar{h}_{1} & =-w_{2} \bar{h}_{2}, \\
\bar{w}_{1} t_{1}+\bar{w}_{2} t_{2} & =w_{1} \bar{t}_{1}+w_{2} \overline{t_{2}}, \quad 0 \leq t_{1}<w_{1}, 0 \leq t_{2}<w_{2}
\end{aligned}
$$

(where the bar denotes conjugation in $\mathbb{Q}(\sqrt{d})$.)

Conversely, let $S$ be a quadratic surface in $\mathcal{H}(2)$. Suppose there exists a direction $v$ in which there is a cylinder decomposition for which the heights, widths and twists (along some completely periodic direction $w$ ) belong to $\mathbb{Q}(\sqrt{d})$ and satisfy (5.4) and (5.5). Then $S$ is Veech.

Proof. First suppose that $S$ is Veech so that $S$ is quadratic. Without loss of generality, we may assume that $v$ is the horizontal direction and $w$ is the vertical direction since application by an element of $S L(2, \mathbb{R})$ conjugates a surface's Veech group by that element, hence preserves the fact that a surface is Veech. Then Theorem 4.2 implies that $S$ has Property X. Since $S$ has Property X, Corollary 4.2 implies that (5.4) and (5.5) hold.

Now assume that the surface $S$ has a horizontal cylinder decomposition for which the widths, heights, and twists (measured along the vertical direction, which is also periodic) belong to $\mathbb{Q}(\sqrt{d})$ and satisfy (5.4) and (5.5). Then Corollary 4.2 implies that $S$ has Property X. But then Theorem 5.1 implies that $S$ is Veech.

Let $\Omega$ be a set containing one element for each Veech surface in $\mathcal{H}(2)$, where we identify any two surfaces in the same $G L(2, \mathbb{R})$ orbit. Let $P$ denote the set of positive solutions in algebraic integers in $\mathbb{Q}(\sqrt{d})$ to (5.4) and (5.5). Then in view of Theorem 5.2 there exists a map $f_{1}: P \rightarrow \Omega$. Now if $S$ is a Veech surface, then there is a surface $S^{\prime}$ in the $G L(2, \mathbb{R})$ orbit of $S$ such that $p\left(H_{1}\left(S^{\prime}\right)\right)$ is a primitive sublattice of $\mathcal{O}_{d} \times \mathcal{O}_{d}$ with no common factor. The area of $S^{\prime}$ is independent of the choice of $S^{\prime}$. Let $f_{2}: \Omega \rightarrow \mathcal{O}_{d}^{+}$denote the map which sends (the equivalence class of) $S$ to the area of $S^{\prime}$.

Corollary 5.4 (Classification of Veech surfaces in $\mathcal{H}(2)$ ). The maps $f_{1}: P \rightarrow \Omega$ and $f_{2}: \Omega \rightarrow \mathcal{O}_{d}^{+}$are finite-to-one.

\section{The Classification OF COMPLETEly PERIODIC SURFACES IN GENUS 2}

We will begin by proving a lemma regarding completely periodic surfaces in the stratum $\mathcal{H}(1,1)$. Then, combining these with the results from the previous section on surfaces in $\mathcal{H}(2)$, we will obtain a classification of all completely periodic translation surfaces in genus two.

First, we will show that if a surface $S \in \mathcal{H}(1,1)$ has a cylinder decomposition in some direction $v$, then applying a map which changes the relative homology of the surface, i.e., moves one singularity with respect to the other but fixes the absolute homology, results in a surface which still has a cylinder decomposition in the direction $v$.

Let $S \in \mathcal{H}(1,1)$. By definition, $S$ has two conical singularities and we may triangulate $S$ via saddle connections to obtain a representation of $S$ as a finite union of polygons in the plane $\mathbb{R}^{2}$, glued along parallel sides which are saddle connections. Consider one of the singularities of the surface $S$ and its images in the polygonal representation of the surface in $\mathbb{R}^{2}, p_{0}, \ldots, p_{n}$. Let $q_{0}, \ldots, q_{m}$ be the images of the second singularity. Given a vector $r \in \mathbb{R}^{2}$, we can form a new translation surface 


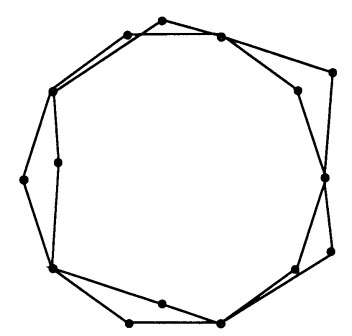

FigURE 8. A perturbed decagon superimposed on a regular decagon.

$S_{r}$ in the following manner. Add $r$ to each of the vertices $p_{0}, \ldots, p_{n}$ while holding fixed the vertices $q_{0}, \ldots, q_{m}$. Let the edges of $S_{r}$ be the line segments of the form $\left[p_{i}+r, q_{j}\right],\left[p_{i}+r, p_{j}+r\right]$, and $\left[q_{i}, q_{j}\right]$ if, respectively, $\left[p_{i}, q_{j}\right],\left[p_{i}, p_{j}\right]$, and $\left[q_{i}, q_{j}\right]$ were edges of $S$. Then we may again glue opposite sides to form a translation surface $S_{r}$. Notice that the map described, which we will refer to as a perturbation of $S$, fixes the absolute homology of $S$ while changing its relative homology.

As a concrete example, we consider the regular decagon, $S_{0}$, with opposite sides identified and having vertices at the points $\left(\cos \left(\frac{k \pi}{5}\right), \sin \left(\frac{k \pi}{5}\right)\right)$ for $k=0, \ldots, 9$. Furthermore, there are two equivalence classes of vertices under the identification correpsonding to $k$ even and $k$ odd. Computing the Euler characteristic of $S_{0}$, we find that $\chi\left(S_{0}\right)=V-E+F=2-5+1=-2$. Therefore the genus of $S_{0}$ is 2 . The two conical singularities of the surface, corresponding to the two equivalence classes of vertices in $S_{0}$, each have total angle $4 \pi$. Thus $S_{0} \in \mathcal{H}(1,1)$. Note that $S_{0}$ is Veech [V1].

To form a perturbed decagon, $S_{r}$, fix one equivalence class of vertices ( $k$ even in Figure 8) while adding the same vector $r$ to each vertex in the other equivalence class ( $k$ odd in Figure 8).

We claim that $S_{r}$ is completely periodic but not Veech. First, we show that $S_{r}$ is not Veech. Since $S_{0}$ is Veech, the moduli of the cylinders in any cylinder decomposition of the surface are rationally commensurate, where the modulus of a cylinder $C_{i}$ is $w_{i} / h_{i}$ [V1]. (Recall that the width denotes the length of the periodic trajectory.) Since $S_{0}$ has a vertical cylinder decomposition, so does $S_{r}$. For small $r$, the vertical cylinders of $S_{r}$ have the same width as those of $S_{0}$ but different heights. A computation shows that the moduli of these cylinders are no longer rationally commensurate. Then $S_{r}$ cannot be Veech [V1].

To see that $S_{r}$ is nonetheless completely periodic, note that since $S_{0}$ is completely periodic (because it is Veech, [V1]) and since the absolute homology of $S_{r}$ is the same as that of $S_{0}$ (although the relative homology changes), $S_{r}$ also has Property $\mathrm{X}$. Thus Theorem 4.1 implies that $S_{r}$ is also completely periodic.

Now consider the set $P=\left\{g S_{r}: g \in S L(2, \mathbb{R}), r \in \mathbb{R}^{2}\right\}$. Note that each surface in $P$ is completely periodic. Then Theorem 6.1 implies that $P$ is a closed, invariant subset of $\mathcal{H}(1,1)$.

Lemma 6.1. Let $S \in \mathcal{H}(1,1)$ be completely periodic, and let $S_{r}$ denote a translation surface obtained from $S$ by changing the relative homology of $S$ while fixing its absolute homology. Then $S_{r}$ is completely periodic as well.

Proof. Since $S$ is completely periodic, Theorem 4.2 implies $S$ has Property X, and by definition, $J$ vanishes in any homological direction. Since $S_{r}$ is obtained from 
$S$ by a map which fixes the absolute homology of the surface, the homological directions of $S_{r}$ are precisely those of $S$. Furthermore, since the $J$-invariant can be calculated in terms of the absolute homology of the surface, $J_{v v}(S)=J_{v v}\left(S_{r}\right)$ for any homological direction $v$. (Note that this implies that if $S$ has a cylinder decomposition in the direction $v$, then $S_{r}$ does as well.) Thus $S_{r}$ has Property X as well. But Theorem 4.1 implies that $S_{r}$ is completely periodic.

Suppose $v$ is a completely periodic direction for $S \in \mathcal{H}(1,1)$. Without loss of generality, we may assume that in the direction $v, S$ decomposes into three cylinders, and for $1 \leq i \leq 3$, let $w_{i}, h_{i}$ and $t_{i}$ denote the widths, heights and twists. After renumbering, we may assume that $w_{3}=w_{1}+w_{2}$. Let $s_{i}=h_{i}+h_{3}$ and $\tau_{i}=t_{i}+t_{3}$ for $i=1,2$. For $c_{1}+c_{2} \sqrt{d} \in \mathcal{O}_{d}^{+}$, consider the following equations:

$$
\begin{aligned}
w_{1} \bar{s}_{1} & =-w_{2} \bar{s}_{2}, \\
\bar{w}_{1} \tau_{1}+\bar{w}_{2} \tau_{2} & =w_{1} \bar{\tau}_{1}+w_{2} \bar{\tau}_{2}, \quad 0 \leq t_{1}<w_{1}, 0 \leq t_{2}<w_{2}, \\
w_{1} s_{1}+w_{2} s_{2} & =2\left(c_{1}+c_{2} \sqrt{d}\right) .
\end{aligned}
$$

Theorem 6.1. Let $\mathcal{M}\left(c_{1}, c_{2}\right)$ denote the set of surfaces in $\mathcal{H}(1,1)$ which are completely periodic and which, for each completely periodic direction $v$, can be rescaled so that the widths, heights and twists of the cylinder decomposition in that direction satisfy (6.1), (6.2) and (6.3). Then $\mathcal{M}\left(c_{1}, c_{2}\right)$ is a closed subset of dimension 5 which is invariant under the $S L(2, \mathbb{R})$ action. It is nonempty provided (6.1), (6.2), and (6.3) have a solution in $\mathcal{O}_{d}^{+}$, and any Veech surface in $\mathcal{H}(1,1)$ is contained in such a subset.

The proof of Theorem 6.1 relies on the the following lemma.

Lemma 6.2. Let $D(t)=\operatorname{diag}\left(t, t, t^{-1}, t^{-1}, t, t\right)$ and let $\Delta$ be a finite set in $\mathbb{R}^{6}$. Let $\mathcal{M}_{2}(\Delta)$ denote the set of completely periodic sufaces in $\mathcal{H}(1,1)$ such that the cylinder decomposition along any periodic direction $v$ satisfies $\left(w_{1}, w_{2}, s_{1}, s_{2}, \tau_{1}, \tau_{2}\right) \in$ $D(\mathbb{R}) \Delta$. Then, provided $\mathcal{M}_{2}(\Delta)$ is nonempty, $\mathcal{M}_{2}(\Delta)$ is a closed invariant subset of $\mathcal{H}(1,1)$.

Proof. The same argument as was used in Lemma 5.2 shows that $\mathcal{M}_{2}(\Delta)$ is invariant under the action of $S L(2, \mathbb{R})$ but with $h_{i}$ and $t_{i}$ replaced by $s_{i}$ and $\tau_{i}$.

Now we must show that $\mathcal{M}_{2}(\Delta)$ is a closed subset of $\mathcal{H}(1,1)$. The proof is similar to that of Lemma 5.2 but with one difference. Suppose that we have a sequence of surfaces $\left\{S_{i}\right\} \subset \mathcal{M}_{2}(\Delta)$ which converges to a surface $S \in \mathcal{H}(1,1)$. As before, we wish to show that $S \in \mathcal{M}_{2}(\Delta)$. In the proof of Lemma 5.2 we argued that if each of the converging surfaces $S_{i} \in \mathcal{M}_{1}(\Delta)$ had a two-cylinder decomposition along a periodic direction $v_{i}$, then the limit surface $S$ would also have a two-cylinder decomposition in a completely periodic direction. However, in this case, since the converging surfaces $S_{i}$ are in $\mathcal{H}(1,1)$, it is possible for a sequence of heights $h_{i}^{j} \rightarrow 0$ for some $j=1,2$ or 3 . We will instead argue that it is not possible for $s_{i}^{j} \rightarrow 0$ for $j=1,2$. Thus it is possible that even if each $S_{i}$ has a three-cylinder decomposition in the completely periodic direction $v_{i}$, the limit surface $S$ could have a two-cylinder decomposition in a completely periodic direction $v$.

In order to show $S$ is completely periodic, we will show that if $S$ has a cylinder with core curve $\gamma$ in some direction $v$, then $S$ has a complete cylinder decomposition in that direction. For large enough $i$, there is a cylinder with core curve $\gamma_{i}$ on each surface $S_{i}$ in some direction $v_{i}$ such that $l_{S_{i}}\left(\gamma_{i}\right) \rightarrow l_{S}(\gamma)=w_{S}^{k}$ and $v_{i} \rightarrow v$. Define 
$w_{i}^{k}=l_{S_{i}}\left(\gamma_{i}\right)$. Since each $S_{i}$ is completely periodic and lies in $\mathcal{M}_{2}(\Delta)$, it has either a two- or three-cylinder decomposition in the direction $v_{i}$. We will assume that $k=1$. Note that if $k=2$, the argument is the same and if $k=3$ so that $w_{i}^{3}=w_{i}^{1}+w_{i}^{2}$, a similar argument gives the result. Also, let $s_{i}^{j}$ and $\tau_{i}^{j}$ for $j=1,2$ be the corresponding parameters associated to the cylinder decomposition of $S_{i}$ in the direction $v_{i}$. Since $\Delta$ is a finite set, we may pass to a subsequence where $w_{i}^{j}=m_{i} w^{j}, s_{i}^{j}=m_{i}^{-1} s^{j}, \tau_{i}^{j}=m_{i} \tau^{j}$ and $\left(w^{1}, w^{2}, s^{1}, s^{2}, \tau^{1}, \tau^{2}\right)$ is a vector in $\Delta$. Since $w_{i}^{1}=m_{i} w^{1} \rightarrow w_{S}^{1}$, we know that $m_{i} \rightarrow m$ and $m \neq 0$. Thus, $w_{i}^{2} \rightarrow m w^{2}$, $s_{i}^{j} \rightarrow m^{-1} s^{j}$, and $\tau_{i}^{j} \rightarrow m \tau^{j}$. In particular, since $m \neq 0, m^{-1} s^{j} \neq 0$. Now recall that the proof of Lemma 6.1 implies that if we apply a map on the relative homology to a surface in $\mathcal{M}_{2}(\Delta)$ which fixes its absolute homology, then the resulting surface is also completely periodic and the parameters $w_{i}, s_{i}, \tau_{i}$ for $i=1,2$ remain stable through the perturbation. Thus $S \in \mathcal{M}_{2}(\Delta)$. Using the convergence, the fact that $m^{-1} s^{j} \neq 0$, and the fact that a cylinder decomposition on each $S_{i}$ is stable under perturbations, we see that $S$ has a cylinder decomposition in the direction $v$ with parameters $m w^{j}, m^{-1} s^{j}, m \tau^{j}$ for $j=1,2$. Thus $\mathcal{M}_{2}(\Delta)$ is closed.

We return to the proof of Theorem 6.1

Proof. The theorem follows mainly from Lemma 6.2 so we need to show that we can apply the lemma. If $S \in \mathcal{M}\left(c_{1}, c_{2}\right)$, then the parameters $w_{i}, s_{i}$ and $\tau_{i}$ for any cylinder decomposition of $S$ must satisfy (6.1), (6.2), and (6.3). Let $\Delta$ be the set of all vectors $\bar{v} \in\left(O_{d}{ }^{+}\right)^{6}$ that satisfy 6.1), 6.2), and 6.3). Now Lemma 5.1 implies there are only finitely many solutions to these equations in $\mathcal{O}_{d}^{+}$, up to the action by units. So $\Delta$ is a finite set. Thus we may apply Lemma 6.2 with $\mathcal{M}_{2}(\Delta)=\mathcal{M}\left(c_{1}, c_{2}\right)$. So $\mathcal{M}\left(c_{1}, c_{2}\right)$ is a closed invariant subset. If $S \in \mathcal{H}(1,1)$ is Veech, it is completely periodic. Theorem 4.2 implies that $S$ has Property X, and so Corollary 4.2 shows that (6.1), (6.2), and (6.3) are satisfied for any cylinder decomposition on $S$. So any Veech surface in $\mathcal{H}(1,1)$ is contained in some $\mathcal{M}\left(c_{1}, c_{2}\right)$.

We also obtain a classification of the completely periodic surfaces in genus two. In fact

Theorem 6.2. In $\mathcal{H}(2)$ every completely periodic surface is Veech. In $\mathcal{H}(1,1)$, a surface is completely periodic if it can be rescaled so that it has a cylinder decomposition in some direction $v$, such that the heights, widths and twists (measured in a completely periodic direction) satisfy (6.1) and (6.2). Conversely, if $S \in \mathcal{H}(1,1)$ is completely periodic, then after rescaling either $p\left(H_{1}(S)\right) \subset \mathbb{Q} \times \mathbb{Q}$ or $p\left(H_{1}(S)\right) \subset \mathbb{Q}(\sqrt{d}) \times \mathbb{Q}(\sqrt{d})$ for some square-free $d>0$. If $S$ cannot be rescaled so that $p\left(H_{1}(S)\right) \subset \mathbb{Q} \times \mathbb{Q}$ and if $v$ is any completely periodic direction, then (6.1) and (6.2) hold.

Proof. If a surface $S \in \mathcal{H}(2)$ is completely periodic, Theorem 4.3 implies that $S$ is quadratic. We apply Theorem 4.2 to conclude that $S$ has Property X. Since $S \in \mathcal{H}(2)$ has Property X, Theorem 5.1 implies that $S$ is Veech.

Now suppose $S \in \mathcal{H}(1,1)$ has a cylinder decomposition in some direction $v$ such that the heights, widths, and twists satisfy (6.1) and (6.2) . Corollary 4.2 implies that $S$ has Property X, and Theorem 4.1 proves that $S$ must be completely periodic. Now, if $S \in \mathcal{H}(1,1)$ is completely periodic, Theorem 4.3 implies that $S$ must be quadratic and Theorem 4.2 shows that $S$ has Property X. If $S$ cannot be rescaled 
so that $p\left(H_{1}(S, \mathbb{Z})\right) \subset \mathbb{Q} \times \mathbb{Q}$ and if $v$ is any completely periodic direction, then Corollary 4.2 proves that (6.1) and (6.2) are satisified.

\section{APPENDIX}

In the appendix, we provide the proofs of several lemmas and theorems which appear throughout the paper.

Lemma 2.2. Let $S \in \mathcal{H}(2)$ and assume that $S$ has a one-cylinder decomposition in the horizontal direction. Then

$$
J(S)=2\left(\left(\begin{array}{c}
w \\
0
\end{array}\right) \wedge\left(\begin{array}{c}
t \\
h
\end{array}\right)+\left(\begin{array}{c}
l_{1} \\
0
\end{array}\right) \wedge\left(\begin{array}{c}
l_{2} \\
0
\end{array}\right)+\left(\begin{array}{c}
l_{2} \\
0
\end{array}\right) \wedge\left(\begin{array}{c}
w \\
0
\end{array}\right)\right)
$$

where $l_{1}$ and $l_{2}$ are the lengths of certain horizontal saddle connections as in Figure 9 If $S \in \mathcal{H}(1,1)$ has a horizontal one-cylinder decomposition, then

$$
J(S)=2\left(\left(\begin{array}{c}
l_{1} \\
0
\end{array}\right) \wedge\left(\begin{array}{c}
l_{2} \\
0
\end{array}\right)+\left(\begin{array}{c}
l_{1}+l_{2} \\
0
\end{array}\right) \wedge\left(\begin{array}{c}
l_{3} \\
0
\end{array}\right)+\left(\begin{array}{c}
l_{2}+l_{3} \\
0
\end{array}\right) \wedge\left(\begin{array}{c}
w \\
0
\end{array}\right)+\left(\begin{array}{c}
w \\
0
\end{array}\right) \wedge\left(\begin{array}{l}
t \\
h
\end{array}\right)\right)
$$

where $l_{1}, l_{2}, l_{3}$ are the lengths of certain horizontal saddle connections as in Figure 10. Now suppose that $S \in \mathcal{H}(2)$ has a two-cylinder decomposition in the horizontal direction. Then the $J$-invariant for $S$ is

$$
J(S)=2\left(\left(\begin{array}{c}
w_{1} \\
0
\end{array}\right) \wedge\left(\begin{array}{c}
t_{1} \\
h_{1}
\end{array}\right)+\left(\begin{array}{c}
w_{2} \\
0
\end{array}\right) \wedge\left(\begin{array}{c}
t_{2} \\
h_{2}
\end{array}\right)\right) .
$$

If $S \in \mathcal{H}(1,1)$ has a two- or three-cylinder decomposition in the horizontal direction, then the $J$-invariant is given by

$$
J(S)=2\left(\left(\begin{array}{c}
w_{1} \\
0
\end{array}\right) \wedge\left(\begin{array}{c}
\tau_{1} \\
s_{1}
\end{array}\right)+\left(\begin{array}{c}
w_{2} \\
0
\end{array}\right) \wedge\left(\begin{array}{c}
\tau_{2} \\
s_{2}
\end{array}\right)\right)
$$

where the cylinders are numbered so that $w_{3}=w_{2}+w_{1}$, and we define $\tau_{i}=t_{i}+t_{3}$, and $s_{i}=h_{i}+h_{3}$ for $i=1,2$.

Proof. First, assume that $S \in \mathcal{H}(2)$ has a one-cylinder decomposition in the horizontal direction. Let $w, h$ and $t$ be the width, height, and twist of this cylinder. Then we can realize $S$ as in Figure 9 Since $S$ consists of a single cylinder of closed horizontal trajectories, the sides of the parallelogram must be identified. Note that since $S \in \mathcal{H}(2)$, it has one singularity of total angle $6 \pi$. Since the top and bottom of the parallelogram must consist of closed, horizontal saddle connections and since the total angle of the singularity is $6 \pi$, there must be three saddle connections, each appearing once on both the top and bottom of the parallelogram. 


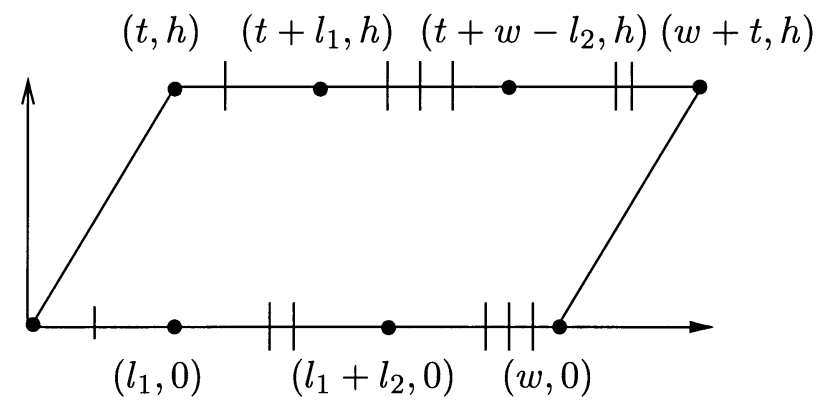

Figure 9. A one-cylinder decomposition of a surface $S \in \mathcal{H}(2)$

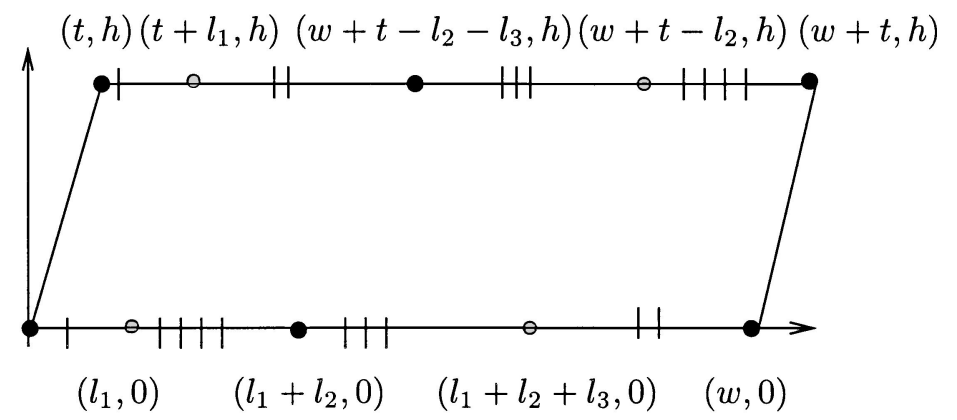

Figure 10. A horizontal one-cylinder decomposition of a surface $S \in \mathcal{H}(1,1)$

Recall that the $J$-invariant is given by $J(S)=\sum_{i=0}^{n} v_{i} \wedge v_{i+1}$ where the $v_{i}$ are the vertices or marked points of the planar representation of $S$, listed in counterclockwise order. So,

$$
\begin{aligned}
J(S) & =\left(\begin{array}{l}
0 \\
0
\end{array}\right) \wedge\left(\begin{array}{c}
l_{1} \\
0
\end{array}\right)+\left(\begin{array}{c}
l_{1} \\
0
\end{array}\right) \wedge\left(\begin{array}{c}
l_{1}+l_{2} \\
0
\end{array}\right)+\left(\begin{array}{c}
l_{1}+l_{2} \\
0
\end{array}\right) \wedge\left(\begin{array}{l}
w \\
0
\end{array}\right)+\left(\begin{array}{c}
w \\
0
\end{array}\right) \wedge\left(\begin{array}{c}
w+t \\
h
\end{array}\right) \\
& +\left(\begin{array}{c}
w+t \\
h
\end{array}\right) \wedge\left(\begin{array}{c}
w+t-l_{2} \\
h
\end{array}\right)+\left(\begin{array}{c}
w+t-l_{2} \\
h
\end{array}\right) \wedge\left(\begin{array}{c}
t+l_{1} \\
h
\end{array}\right) \\
& +\left(\begin{array}{c}
t+l_{1} \\
h
\end{array}\right) \wedge\left(\begin{array}{l}
t \\
h
\end{array}\right)+\left(\begin{array}{l}
t \\
h
\end{array}\right) \wedge\left(\begin{array}{l}
0 \\
0
\end{array}\right) \\
& =\left(\begin{array}{c}
l_{1} \\
0
\end{array}\right) \wedge\left(\begin{array}{c}
l_{2} \\
0
\end{array}\right)+\left(\begin{array}{c}
l_{1} \\
0
\end{array}\right) \wedge\left(\begin{array}{c}
w \\
0
\end{array}\right)+\left(\begin{array}{c}
l_{2} \\
0
\end{array}\right) \wedge\left(\begin{array}{c}
w \\
0
\end{array}\right)+\left(\begin{array}{c}
w \\
0
\end{array}\right) \wedge\left(\begin{array}{c}
t \\
h
\end{array}\right) \\
& -\left(\begin{array}{c}
w+t \\
h
\end{array}\right) \wedge\left(\begin{array}{c}
l_{2} \\
0
\end{array}\right)+\left(\begin{array}{c}
w-l_{2} \\
0
\end{array}\right) \wedge\left(\begin{array}{c}
l_{1} \\
0
\end{array}\right)+\left(\begin{array}{c}
w-l_{2} \\
0
\end{array}\right) \wedge\left(\begin{array}{c}
t \\
h
\end{array}\right) \\
& +\left(\begin{array}{c}
t \\
h
\end{array}\right) \wedge\left(\begin{array}{c}
l_{1} \\
0
\end{array}\right)+\left(\begin{array}{c}
l_{1} \\
0
\end{array}\right) \wedge\left(\begin{array}{c}
t \\
h
\end{array}\right) .
\end{aligned}
$$

Simplifying, we find that

$$
J(S)=2\left(\left(\begin{array}{c}
w \\
0
\end{array}\right) \wedge\left(\begin{array}{c}
t \\
h
\end{array}\right)+\left(\begin{array}{c}
l_{1} \\
0
\end{array}\right) \wedge\left(\begin{array}{c}
l_{2} \\
0
\end{array}\right)+\left(\begin{array}{c}
l_{2} \\
0
\end{array}\right) \wedge\left(\begin{array}{c}
w \\
0
\end{array}\right)\right) .
$$




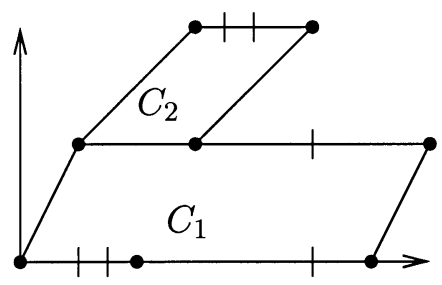

Figure 11. A horizontal two-cylinder decomposition of a surface $S \in \mathcal{H}(2)$

If $S \in \mathcal{H}(1,1)$ has a horizontal one-cylinder decomposition, then we can calculate its $J$-invariant in a similar way. Referring to Figure 10, we have

$$
\begin{aligned}
J(S) & =\left(\begin{array}{c}
l_{1} \\
0
\end{array}\right) \wedge\left(\begin{array}{c}
l_{1}+l_{2} \\
0
\end{array}\right)+\left(\begin{array}{c}
l_{1}+l_{2} \\
0
\end{array}\right) \wedge\left(\begin{array}{c}
l_{1}+l_{2}+l_{3} \\
0
\end{array}\right)+\left(\begin{array}{c}
l_{1}+l_{2}+l_{3} \\
0
\end{array}\right) \wedge\left(\begin{array}{c}
w \\
0
\end{array}\right) \\
& +\left(\begin{array}{c}
w \\
0
\end{array}\right) \wedge\left(\begin{array}{c}
w+t \\
h
\end{array}\right)+\left(\begin{array}{c}
w+t \\
h
\end{array}\right) \wedge\left(\begin{array}{c}
w+t-l_{2} \\
h
\end{array}\right) \\
& +\left(\begin{array}{c}
w+t-l_{2} \\
h
\end{array}\right) \wedge\left(\begin{array}{c}
w+t-l_{2}-l_{3} \\
h
\end{array}\right)+\left(\begin{array}{c}
w+t-l_{2}-l_{3} \\
h
\end{array}\right) \wedge\left(\begin{array}{c}
t+l_{1} \\
h
\end{array}\right) \\
& +\left(\begin{array}{c}
t+l_{1} \\
h
\end{array}\right) \wedge\left(\begin{array}{l}
t \\
h
\end{array}\right) .
\end{aligned}
$$

Simplifying, we have that

$$
\begin{aligned}
J(S) & =\left(\begin{array}{c}
l_{1} \\
0
\end{array}\right) \wedge\left(\begin{array}{c}
l_{2} \\
0
\end{array}\right)+\left(\begin{array}{c}
l_{1} \\
0
\end{array}\right) \wedge\left(\begin{array}{c}
l_{3} \\
0
\end{array}\right)+\left(\begin{array}{c}
l_{2} \\
0
\end{array}\right) \wedge\left(\begin{array}{c}
l_{3} \\
0
\end{array}\right)+\left(\begin{array}{c}
l_{1} \\
0
\end{array}\right) \wedge\left(\begin{array}{c}
w \\
0
\end{array}\right)+\left(\begin{array}{c}
l_{2} \\
0
\end{array}\right) \wedge\left(\begin{array}{c}
w \\
0
\end{array}\right) \\
& +\left(\begin{array}{c}
l_{3} \\
0
\end{array}\right) \wedge\left(\begin{array}{c}
w \\
0
\end{array}\right)+\left(\begin{array}{c}
w \\
0
\end{array}\right) \wedge\left(\begin{array}{c}
t \\
h
\end{array}\right)+\left(\begin{array}{c}
w+t \\
h
\end{array}\right) \wedge\left(\begin{array}{c}
-l_{2} \\
0
\end{array}\right)+\left(\begin{array}{c}
w+t-l_{2} \\
h
\end{array}\right) \wedge\left(\begin{array}{c}
-l_{3} \\
0
\end{array}\right) \\
& +\left(\begin{array}{c}
w-l_{2}-l_{3} \\
0
\end{array}\right) \wedge\left(\begin{array}{c}
t \\
h
\end{array}\right)+\left(\begin{array}{c}
w-l_{2}-l_{3} \\
0
\end{array}\right) \wedge\left(\begin{array}{c}
l_{1} \\
0
\end{array}\right)+\left(\begin{array}{c}
t \\
h
\end{array}\right) \wedge\left(\begin{array}{c}
l_{1} \\
0
\end{array}\right) .
\end{aligned}
$$

Further computation shows that

$J(S)=2\left(\left(\begin{array}{c}l_{1} \\ 0\end{array}\right) \wedge\left(\begin{array}{c}l_{2} \\ 0\end{array}\right)+\left(\begin{array}{c}l_{1}+l_{2} \\ 0\end{array}\right) \wedge\left(\begin{array}{c}l_{3} \\ 0\end{array}\right)+\left(\begin{array}{c}l_{2}+l_{3} \\ 0\end{array}\right) \wedge\left(\begin{array}{c}w \\ 0\end{array}\right)+\left(\begin{array}{c}w \\ 0\end{array}\right) \wedge\left(\begin{array}{l}t \\ h\end{array}\right)\right)$.

Now suppose that $S \in \mathcal{H}(2)$ has a two-cylinder decomposition in the horizontal direction. As before, we can realize $S$ as the union of two parallelograms or cylinders of closed horizontal trajectories with parallel sides identified as in Figure 11.

In order to calculate $J(S)$, it suffices to calculate the $J$-invariant of each of the two cylinders $C_{1}$ and $C_{2}$ separately and then add them together. Since $J$ is translation invariant, we can picture each of the cylinders as having its bottom left endpoint at the origin. Since $C_{2}$ is a parallelogram whose only marked points occur at each of its four corners, Lemma 2.1 implies that

$$
J\left(C_{2}\right)=2\left(\left(\begin{array}{c}
w_{2} \\
0
\end{array}\right) \wedge\left(\begin{array}{c}
t_{2} \\
h_{2}
\end{array}\right)\right)
$$




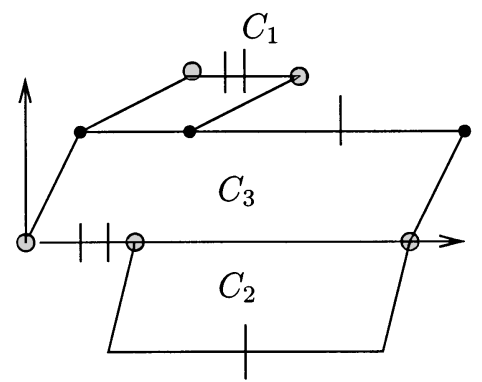

Figure 12. A horizontal three-cylinder decomposition of a surface $S \in \mathcal{H}(1,1)$

and by definition,

$$
\begin{aligned}
J\left(C_{1}\right) & =\left(\begin{array}{c}
0 \\
0
\end{array}\right) \wedge\left(\begin{array}{c}
w_{2} \\
0
\end{array}\right)+\left(\begin{array}{c}
w_{2} \\
0
\end{array}\right) \wedge\left(\begin{array}{c}
w_{1} \\
0
\end{array}\right)+\left(\begin{array}{c}
w_{1} \\
0
\end{array}\right) \wedge\left(\begin{array}{c}
w_{1}+t_{1} \\
h_{1}
\end{array}\right) \\
& +\left(\begin{array}{c}
w_{1}+t_{1} \\
h_{1}
\end{array}\right) \wedge\left(\begin{array}{c}
w_{2}+t_{1} \\
h_{1}
\end{array}\right)+\left(\begin{array}{c}
w_{2}+t_{1} \\
h_{1}
\end{array}\right) \wedge\left(\begin{array}{c}
t_{1} \\
h_{1}
\end{array}\right)+\left(\begin{array}{c}
t_{1} \\
h_{1}
\end{array}\right) \wedge\left(\begin{array}{l}
0 \\
0
\end{array}\right) \\
& =\left(\begin{array}{c}
w_{2} \\
0
\end{array}\right) \wedge\left(\begin{array}{c}
w_{1} \\
0
\end{array}\right)+\left(\begin{array}{c}
w_{1} \\
0
\end{array}\right) \wedge\left(\begin{array}{c}
t_{1} \\
h_{1}
\end{array}\right)+\left(\begin{array}{c}
w_{1} \\
0
\end{array}\right) \wedge\left(\begin{array}{c}
w_{2} \\
0
\end{array}\right) \\
& +\left(\begin{array}{c}
w_{1} \\
0
\end{array}\right) \wedge\left(\begin{array}{c}
t_{1} \\
h_{1}
\end{array}\right)+\left(\begin{array}{c}
t_{1} \\
h_{1}
\end{array}\right) \wedge\left(\begin{array}{c}
w_{2} \\
0
\end{array}\right)+\left(\begin{array}{c}
w_{2} \\
0
\end{array}\right) \wedge\left(\begin{array}{c}
t_{1} \\
h_{1}
\end{array}\right) \\
& =2\left(\left(\begin{array}{c}
w_{1} \\
0
\end{array}\right) \wedge\left(\begin{array}{c}
t_{1} \\
h_{1}
\end{array}\right)\right) .
\end{aligned}
$$

Upon adding $J\left(C_{1}\right)$ and $J\left(C_{2}\right)$, we find that

$$
J(S)=J\left(C_{1}\right)+J\left(C_{2}\right)=2\left(\left(\begin{array}{c}
w_{1} \\
0
\end{array}\right) \wedge\left(\begin{array}{c}
t_{1} \\
h_{1}
\end{array}\right)+\left(\begin{array}{c}
w_{2} \\
0
\end{array}\right) \wedge\left(\begin{array}{c}
t_{2} \\
h_{2}
\end{array}\right)\right) .
$$

Now suppose that $S \in \mathcal{H}(1,1)$ has a three-cylinder decomposition in the horizontal direction. Then $S$ consists of two cylinders each of which is bounded on either side by a closed saddle connection connecting one singularity to itself and a third cylinder whose boundary consists of the saddle connections which form the boundaries of the other two cylinders. Thus, the third cylinder has two closed saddle connections connecting one singularity to itself on one side and another two closed saddle connections connecting the other singularity to itself on the other side. If $w_{1}$ and $w_{2}$ are the widths of the first two cylinders, $C_{1}$ and $C_{2}$, then the width of the third, wider, cylinder, $C_{3}$, is $w_{3}=w_{1}+w_{2}$. So we can represent $S$ as the union of three parallelograms in the plane with opposite sides identified as in Figure 12.

As before, we can picture each cylinder $C_{i}$ as having its bottom left corner at the origin and we can calculate $J(S)$ using the formula $J(S)=J\left(C_{1}\right)+J\left(C_{2}\right)+J\left(C_{3}\right)$. Since $C_{1}$ and $C_{2}$ are cylinders whose only marked points occur at each of their corners, Lemma 2.1 implies that

$$
J\left(C_{i}\right)=2\left(\left(\begin{array}{c}
w_{i} \\
0
\end{array}\right) \wedge\left(\begin{array}{c}
t_{i} \\
h_{i}
\end{array}\right)\right)
$$

for $i=1,2$. It remains to calculate $J\left(C_{3}\right)$. Using Figure 12 and the definition of the $J$-invariant, the same calculation that was used to find the $J$-invariant of a cylinder 
with more than one saddle connection on each boundary for a surface $S \in \mathcal{H}(2)$ shows that

$$
J\left(C_{3}\right)=2\left(\left(\begin{array}{c}
w_{3} \\
0
\end{array}\right) \wedge\left(\begin{array}{c}
t_{3} \\
h_{3}
\end{array}\right)\right) .
$$

Summing over the $J$-invariants of the individual cylinders yields

$$
\begin{aligned}
J(S) & =J\left(C_{1}\right)+J\left(C_{2}\right)+J\left(C_{3}\right) \\
& =2\left(\left(\begin{array}{c}
w_{1} \\
0
\end{array}\right) \wedge\left(\begin{array}{c}
t_{1} \\
h_{1}
\end{array}\right)+\left(\begin{array}{c}
w_{2} \\
0
\end{array}\right) \wedge\left(\begin{array}{c}
t_{2} \\
h_{2}
\end{array}\right)+\left(\begin{array}{c}
w_{3} \\
0
\end{array}\right) \wedge\left(\begin{array}{c}
t_{3} \\
h_{3}
\end{array}\right)\right) .
\end{aligned}
$$

If we let $\tau_{i}=t_{i}+t_{3}$ and $s_{i}=h_{i}+h_{3}$ for $i=1,2$ and recall that $w_{3}=w_{2}+w_{1}$, this yields (7.3).

Finally, consider the case where $S \in \mathcal{H}(1,1)$ and $S$ has a two-cylinder decomposition in the horizontal direction. In fact, we can calculate $J(S)$ in this case exactly as we did for a surface $S \in \mathcal{H}(1,1)$ having a three-cylinder decomposition in the horizontal direction, except that here $h_{3}=0$. Therefore we again find that

$$
J(S)=2\left(\left(\begin{array}{c}
w_{1} \\
0
\end{array}\right) \wedge\left(\begin{array}{c}
\tau_{1} \\
s_{1}
\end{array}\right)+\left(\begin{array}{c}
w_{2} \\
0
\end{array}\right) \wedge\left(\begin{array}{c}
\tau_{2} \\
s_{2}
\end{array}\right)\right) .
$$

Theorem 7.1. Let $S$ be a genus 2 completely periodic translation surface with a one-cylinder decomposition in some direction. Then $S$ can be rescaled so that $p\left(H_{1}(S, \mathbb{Z})\right) \subset \mathbb{Q} \times \mathbb{Q}$.

Proof. First suppose that $S \in \mathcal{H}(2)$ is completely periodic. We will assume without loss of generality that the vertical, horizontal, and $\left(\begin{array}{l}1 \\ 1\end{array}\right)$ directions on $S$ are completely periodic and that there is a one-cylinder decomposition in the horizontal direction. Then the previous lemma implies that

$$
J(S)=2\left(\left(\begin{array}{c}
w \\
0
\end{array}\right) \wedge\left(\begin{array}{l}
t \\
h
\end{array}\right)+\left(\begin{array}{c}
l_{1} \\
0
\end{array}\right) \wedge\left(\begin{array}{c}
l_{2} \\
0
\end{array}\right)+\left(\begin{array}{c}
l_{2} \\
0
\end{array}\right) \wedge\left(\begin{array}{c}
w \\
0
\end{array}\right)\right)
$$

where $w, t, h, l_{1}$ and $l_{2}$ are as in the statement of Lemma 2.2 Since the vertical direction is completely periodic, Theorem 2.2 implies

$$
J_{x x}(S)=2\left(w \wedge t+l_{1} \wedge l_{2}+l_{2} \wedge w\right)=0 .
$$

Also, since the $\left(\begin{array}{l}1 \\ 1\end{array}\right)$ direction is completely periodic,

$$
J_{y y}(g S)=2\left(-w \wedge h+\left(w \wedge t+l_{1} \wedge l_{2}+l_{2} \wedge w\right)\right)=0
$$

where

$$
g=\left(\begin{array}{cc}
1 & 0 \\
-1 & 1
\end{array}\right)
$$

Combining (7.6) and (7.7), we find that

$$
w \wedge h=0 .
$$

Thus $w=r h$ for some $r \in \mathbb{Q}$. We can rescale the surface by dividing each coordinate of each point by $w$ and in so doing will not change the fact that the horizontal, vertical and $\left(\begin{array}{l}1 \\ 1\end{array}\right)$ directions are completely periodic. So we may assume $w=1$, which implies $h \in \mathbb{Q}$. Let $v=\left(\begin{array}{l}1 \\ q\end{array}\right)$ be another completely periodic direction on $S$. Then Theorem 2.2 implies

$$
J_{v v}(S)=J_{y y}\left(g_{q} S\right)=0
$$


where

$$
g_{q}=\left(\begin{array}{cc}
1 & 0 \\
-q & 1
\end{array}\right)
$$

A straightforward calculation shows that (7.8) can be rewritten as

$$
J_{v v}(S)=2\left(-q \wedge h+\left(q \wedge q t+q l_{1} \wedge q l_{2}+q l_{2} \wedge q\right)\right)=0 .
$$

Equation (17.6) implies $q \wedge q t+q l_{1} \wedge q l_{2}+q l_{2} \wedge q=0$ as well. Thus (17.9) yields

$$
q \wedge h=0 \text {. }
$$

Since $h \in \mathbb{Q}$, this implies $q \in \mathbb{Q}$ as well. So any completely periodic direction on $S$ is rational.

The rest of the proof mirrors that of Theorem 4.2 Once again referring to Figure 9, we see that the vectors $\left(\begin{array}{l}t \\ h\end{array}\right),\left(\begin{array}{c}t+1-l_{1}-l_{2} \\ h\end{array}\right)$, and $\left(\begin{array}{c}t-l_{2} \\ h\end{array}\right)$ represent cylinders of closed trajectories, and so their corresponding directions are completely periodic. But the above argument shows that the directions of each of these vectors lies in $\mathbb{Q}$. In other words, there exist $r_{i} \in \mathbb{Q}$ such that

$$
\begin{aligned}
& h=r_{1} t, \\
& h=r_{2}\left(t+1-l_{1}-l_{2}\right), \\
& h=r_{3}\left(t-l_{2}\right) .
\end{aligned}
$$

Since $h \in \mathbb{Q}$, (7.10) implies $t \in \mathbb{Q}$. Given that $t, h \in \mathbb{Q}$, (7.11) implies $l_{1}+l_{2} \in \mathbb{Q}$. Finally, (7.12) shows that $l_{2} \in \mathbb{Q}$ and so $l_{1} \in \mathbb{Q}$ as well. Since each of the cylinder parameters $l_{1}, l_{2}, w, t, h \in \mathbb{Q}$, we have that $p\left(H_{1}(S, \mathbb{Z})\right) \subset \mathbb{Q} \times \mathbb{Q}$.

The case when $S \in \mathcal{H}(1,1)$ is entirely analagous. Recall that Lemma 2.2 implies that

$$
J(S)=2\left(\left(\begin{array}{l}
l_{1} \\
0
\end{array}\right) \wedge\left(\begin{array}{l}
l_{2} \\
0
\end{array}\right)+\left(\begin{array}{c}
l_{1}+l_{2} \\
0
\end{array}\right) \wedge\left(\begin{array}{c}
l_{3} \\
0
\end{array}\right)+\left(\begin{array}{c}
l_{2}+l_{3} \\
0
\end{array}\right) \wedge\left(\begin{array}{l}
w \\
0
\end{array}\right)+\left(\begin{array}{l}
w \\
0
\end{array}\right) \wedge\left(\begin{array}{l}
t \\
h
\end{array}\right)\right)
$$

where all paramters are as in the lemma. The same argument as for the case $S \in \mathcal{H}(2)$ shows that $S$ can be rescaled so that $w=1, h \in \mathbb{Q}$, and if $\left(\begin{array}{l}1 \\ q\end{array}\right)$ is any completely periodic direction, then $q \in \mathbb{Q}$. Referring to Figure 10] we see that the vectors $\left(\begin{array}{l}t \\ h\end{array}\right),\left(\begin{array}{c}t+w-l_{2}-l_{3} \\ h\end{array}\right)$ and $\left(\begin{array}{c}t+w-l_{1}-l_{2} \\ h\end{array}\right)$ represent cylinders of closed trajectories. Hence each of the directions corresponding to these vectors is completely periodic, and since any completely periodic direction is rational, there exist $p_{i} \in \mathbb{Q}$ such that

$$
\begin{aligned}
& h=p_{1} t, \\
& h=p_{2}\left(t+-l_{2}-l_{3}\right), \\
& h=p_{3}\left(t+1-l_{1}-2 l_{2}-l_{3}\right) .
\end{aligned}
$$

As before, since $h \in \mathbb{Q}$, (17.13) implies $t \in \mathbb{Q}$. Then (17.14) implies $l_{2}+l_{3} \in \mathbb{Q}$ and (7.15) implies $l_{1}+l_{2} \in \mathbb{Q}$. Note that, in contrast to the case when $S \in \mathcal{H}(2)$, this only implies that the absolute homology $p\left(H_{1}(S, \mathbb{Z})\right) \subset \mathbb{Q} \times \mathbb{Q}$ but not necessarily the relative homology.

Lemma 2.4. Let the direction $v$ on the translation surface $S$ be represented by the vector $\left(\begin{array}{l}1 \\ q\end{array}\right)$, where $v$ is neither the horizontal nor vertical direction, and suppose that $g \in S L(2, \mathbb{R})$ is any matrix such that $g\left(\begin{array}{l}1 \\ q\end{array}\right)=\left(\begin{array}{l}1 \\ 0\end{array}\right)$. Then $J_{y y}(g S)=J_{y y}\left(g_{q} S\right)$ where

$$
g_{q}=\left(\begin{array}{cc}
1 & 0 \\
-q & 1
\end{array}\right)
$$


Proof. By assumption, $g^{-1} g_{q} \in \operatorname{Stab}_{S L(2, \mathbb{R})}\left(\left(\begin{array}{l}1 \\ q\end{array}\right)\right)$. Now $\operatorname{Stab}_{S L(2, \mathbb{R})}\left(\left(\begin{array}{l}1 \\ q\end{array}\right)\right)=r_{q} N r_{q}^{-1}$ where

$$
r_{q}=\left(\begin{array}{cc}
\cos (\theta) & -\sin (\theta) \\
\sin (\theta) & \cos (\theta)
\end{array}\right)
$$

is the counterclockwise rotation by $\theta=\tan ^{-1}(q)$ and $N$ is the subgroup of uppertriangular unipotent matrices, i.e., the stabilizer of the vector $\left(\begin{array}{l}1 \\ 0\end{array}\right)$. Considering the definition of $J_{y y}(S)$, it suffices to show that if $\left(\begin{array}{l}a \\ b\end{array}\right)$ is an arbitrary vector and $m \in N$, then the $y$-coordinates of $g_{q}\left(\begin{array}{l}a \\ b\end{array}\right)$ and $g_{q} r_{q} m r_{q}{ }^{-1}\left(\begin{array}{l}a \\ b\end{array}\right)$ are equal. First, notice that $g_{q}\left(\begin{array}{l}a \\ b\end{array}\right)=\left(\begin{array}{c}a \\ -q a+b\end{array}\right)$. Let

$$
m=\left(\begin{array}{ll}
1 & n \\
0 & 1
\end{array}\right) .
$$

A straightforward calculation shows that

$$
r_{q} m r_{q}{ }^{-1}=\left(\begin{array}{cc}
1-n \cos (\theta) \sin (\theta) & n \cos ^{2}(\theta) \\
-n \sin ^{2}(\theta) & 1+n \cos (\theta) \sin (\theta)
\end{array}\right) .
$$

Note that we can write

$$
g_{q}=\left(\begin{array}{cc}
1 & 0 \\
-\frac{\sin (\theta)}{\cos (\theta)} & 1
\end{array}\right) \text {. }
$$

Thus,

$$
\begin{aligned}
g_{q} r_{q} m r_{q}^{-1}\left(\begin{array}{l}
a \\
b
\end{array}\right) & =\left(\begin{array}{cc}
1-n \cos (\theta) \sin (\theta) & n \cos ^{2}(\theta) \\
-q & 1
\end{array}\right)\left(\begin{array}{l}
a \\
b
\end{array}\right) \\
& =\left(\begin{array}{c}
a(1-n \cos (\theta) \sin (\theta))+b n \cos ^{2}(\theta) \\
-q a+b
\end{array}\right) .
\end{aligned}
$$

This completes the proof.

Lemma 3.3. If $f$ is an interval exchange transformation on either two or three intervals and if $S A F(f)=0$, then $f$ is periodic.

Proof. We will prove the result for 2-iets and then deduce it for 3-iets by reducing to the 2-iet case. Without loss of generality, we will assume that all iets mentioned are maps of $I=[0,1]$. Let $I^{\prime}$ be another copy of $I$ to be thought of as the range of $f$. Let $0=\lambda_{0}<\lambda_{1}<\cdots<\lambda_{n}=1$ and $0=\lambda_{0}{ }^{\prime}<\lambda_{1}{ }^{\prime}<\cdots<\lambda_{n}{ }^{\prime}=1$ be partitions of the intervals $I$ and $I^{\prime}$, respectively. Let $\sigma$ be a permutation of the set $\{1, \cdots, n\}$. Furthermore, let $I_{j}=\left[\lambda_{j-1}, \lambda_{j}\right]$ and $I_{j}{ }^{\prime}=\left[\lambda_{j-1}{ }^{\prime}, \lambda_{j}{ }^{\prime}\right]$ for $1 \leq j \leq 2$ and assume that the length $l_{j}$ of $I_{j}$ is equal to the length $l_{j}{ }^{\prime}$ of $I_{\sigma(j)}{ }^{\prime}$. Then we define $f:[0,1] \rightarrow[0,1]$ outside the set $\left\{\lambda_{0}, \cdots, \lambda_{n}\right\}$ as $f(x)=x+\tau_{j}$ where $x \in \stackrel{\circ}{I}_{j}$ for some $1 \leq j \leq n$, and $\tau_{j}=\lambda_{\sigma(j)}{ }^{\prime}-\lambda_{j}$ is the amount the interval $\stackrel{\circ}{I_{j}}$ is translated by $f$. Recall that

$$
S A F(f)=\sum_{j=1}^{n} l_{j} \wedge \tau_{j}
$$

First we consider the case when $n=2$. If $f$ is not the identity, in which case $f$ is trivially periodic, then the permutation associated to $f$ is the transposition $(1,2)$. 
In this case, $f(x)=x+1-\lambda_{1}$ for $x \in \stackrel{\circ}{I_{1}}$ and $f(x)=x-\lambda_{1}$ for $x \in \stackrel{\circ}{I_{2}}$. Then $l_{1}=\lambda_{1}, l_{2}=1-\lambda_{1}, \tau_{1}=1-\lambda_{1}$, and $\tau_{2}=-\lambda_{1}$. By definition,

$$
\begin{aligned}
S A F(f) & =\left(\lambda_{1}\right) \wedge\left(1-\lambda_{1}\right)+\left(1-\lambda_{1}\right) \wedge\left(-\lambda_{1}\right) \\
& =\lambda_{1} \wedge 1-1 \wedge \lambda_{1} \\
& =2\left(\lambda_{1} \wedge 1\right) .
\end{aligned}
$$

If $S A F(f)=0$, then $\lambda_{1} \in \mathbb{Q}$. Since $\lambda_{1}$ is rational, $f$ corresponds to a rotation of the circle by a rational multiple of $\pi$; hence it is periodic.

Next we consider the case when $f$ is a 3 -iet. There are five nontrivial possibilities for the permutation $\sigma$ corresponding to $f$. Of these five, four of the permutations, namely, (312), (231), (132) and (213), can be reduced immediately to the 2-iet case. The permutations (312) and (231) can be considered 2-iets by gluing together two contiguous intervals and then transposing that larger interval with the third. The SAF invariant will remain the same. To see this, consider an iet $f$ with permutation (312) and corresponding intervals $I_{i}$ for $i=1,2,3$. Note that in this case, $\tau_{1}=\tau_{2}$. Then $f$ can be reduced to a 2 -iet $g$ with permutation $(1,2)$ and exchanged intervals $J_{1}=I_{1} \cup I_{2}$ and $J_{2}=I_{3}$. We have, by definition and simplification,

$$
\begin{aligned}
S A F(f) & =\Sigma_{i=1}^{3} l_{i} \wedge \tau_{i} \\
& =\left(l_{1}+l_{2}\right) \wedge \tau_{1}+l_{3} \wedge \tau_{3} \\
& =S A F(g) .
\end{aligned}
$$

Since $S A F(f)=0, S A F(g)=0$. But $g$ is a 2-iet, and the previous argument proves $g$ is periodic; hence $f$ is. A similar argument holds for the 3 -iet associated to the permutation (231).

The 3 -iets corresponding to the permutations (132) and (213) reduce to 2 -iets on the two intervals which are transposed. Let $f$ be an iet of $[0,1]$ with permutation (132) and intervals $I_{i}$ for $i=1,2,3$. Lemma 3.2 implies

$$
S A F(f)=S A F\left(f_{I_{1}}\right)+S A F\left(f_{I_{2} \cup I_{3}}\right)
$$

and Theorem 3.2 shows that $S A F\left(f_{I_{1}}\right)=0$. Since $S A F(f)=0$ as well, the above equation shows that $S A F\left(f_{I_{2} \cup I_{3}}\right)=0$. But $f_{I_{2} \cup I_{3}}$ is a 2-iet, so $f_{I_{2} \cup I_{3}}$ is periodic. Since $f_{I_{1}}$ is also periodic (it is the identity), $f$ itself is periodic as well.

We are left to consider the 3 -iet $f$ on the interval $[0,1]$ with partition $\left\{\lambda_{0}=\right.$ $\left.0, \lambda_{1}, \lambda_{2}, \lambda_{3}=1\right\}$ associated to the permutation (321). We will derive a 2 -iet $g$ from $f$ using Rauzy induction on a certain subinterval of $[0,1]$. Since $S A F(\cdot)$ is invariant under Rauzy induction, $S A F(f)=S A F(g)$.

First we will determine the 2 -iet $g$, which will be the first return map to a certain subinterval of $[0,1]$. The argument breaks into two cases depending on whether $l_{3}$ is less than or greater than $l_{1}$. We will assume that $l_{3}>l_{1}$. If $l_{3}<l_{1}$, the proof is similar. Let $g$ be the iet obtained from $f$ by inducing on the subinterval $J=\left[0,1-\lambda_{1}\right]$. Note that the lengths of the subintervals associated to $f$ are $l_{1}=\lambda_{1}, l_{2}=\lambda_{2}-\lambda_{1}, l_{3}=1-\lambda_{2}$, while the translation distances are $\tau_{1}=1-\lambda_{1}$, $\tau_{2}=1-\lambda_{2}-\lambda_{1}$, and $\tau_{3}=-\lambda_{2}$. ( In fact, the expressions for the interval lengths and the translation distances are the same whether $l_{3}>l_{1}$ or $l_{3}<l_{1}$.) Now we will partition $J$ into three subintervals as well using the partition $\left\{\lambda_{0}=0, \lambda_{1}, \lambda_{2}, 1-\lambda_{1}\right\}$. Since $\tau_{1}=1-\lambda_{1}$, the interval $\left(0, \lambda_{1}\right)$ is sent by $f$ to the interval $\left(1-\lambda_{1}, 1\right)$, which is not a subinterval of $J$. However, since $l_{1}<l_{3}$ and $\tau_{3}=-\lambda_{2}, f$ takes the interval $\left(1-\lambda_{1}, 1\right)$ and sends it to the interval $\left(1-\lambda_{1}-\lambda_{2}, 1-\lambda_{2}\right)$, which does lie in $J$. Next 
we consider the second subinterval of $J$, namely $\left(\lambda_{1}, \lambda_{2}\right)$. Note that $f$ sends $\left(\lambda_{1}, \lambda_{2}\right)$ to the interval $\left(1-\lambda_{2}, 1-\lambda_{1}\right)$ since $\tau_{2}=1-\lambda_{2}-\lambda_{1}$. In this case, $\left(1-\lambda_{2}, 1-\lambda_{1}\right)$ is a subinterval of $J$, so the first return map $g$ sends $\left(\lambda_{1}, \lambda_{2}\right)$ to the subinterval $\left(1-\lambda_{2}, 1-\lambda_{1}\right)$. Finally consider the third subinterval of $J,\left(\lambda_{2}, 1-\lambda_{1}\right)$. Since $\tau_{3}=-\lambda_{2}$, this interval is sent by $f$ to the interval $\left(0,1-\lambda_{2}-\lambda_{1}\right)$, which is a subinterval of $J$. This implies that the permutation associated to $g$ is (312).

Since $S A F(f)=S A F(g)=0$ and since the permutation for $g$ is (312), a previous case shows that $g$, hence $f$, is periodic.

Lemma 5.1. For fixed integers $c_{1}$ and $c_{2}$, such that $c_{1}$ is divisible by $d$, the equations

$$
\begin{aligned}
w_{1} \bar{h}_{1} & =-w_{2} \bar{h}_{2}, \\
\bar{w}_{1} t_{1}+\bar{w}_{2} t_{2} & =w_{1} \bar{t}_{1}+w_{2} \bar{t}_{2}, \quad 0 \leq t_{i} \leq w_{i}, \\
w_{1} h_{1}+w_{2} h_{2} & =2\left(c_{1}+c_{2} \sqrt{d}\right)
\end{aligned}
$$

have finitely many solutions in $\mathcal{O}_{d}^{+}$, the positive algebraic integers in $\mathbb{Q}(\sqrt{d})$, up to the action by the group of units. Let us denote this finite number by $H\left(c_{1}, c_{2}\right)$.

Proof. We will assume $d \equiv 2$ or $3 \bmod 4$. If $d \equiv 1 \bmod 4$, the proof is similar. Since $d \equiv 2$ or $3 \bmod 4$, and $w_{i}, h_{i}, t_{i}$ are algebraic integers in $\mathbb{Q}(\sqrt{d})$, we may write $w_{i}=w_{i}^{1}+w_{i}^{2} \sqrt{d}, h_{i}=h_{i}^{1}+h_{i}^{2} \sqrt{d}$, and $t_{i}=t_{i}^{1}+t_{i}^{2} \sqrt{d}$ for $w_{i}^{j}, h_{i}^{j}, t_{i}^{j} \in \mathbb{Z}$. Since we wish to prove that (17.17), (7.18) and (7.19) have finitely many solutions in $\mathcal{O}_{d}^{+}$ up to the action by the group of units, we will show that there are only finitely many solutions which lie in a fundamental domain for the action of the group of units on $\mathcal{O}_{d}^{+}$. In order to describe such a fundamental domain, we will associate to each algebraic integer $x+y \sqrt{d}$, the point $(x, y)$ in the plane. If we let $n$ be the algebraic norm of $x+y \sqrt{d}$, that is, $n=(x+y \sqrt{d})(x-y \sqrt{d})=x^{2}-y^{2} d$, then the set of algebraic integers of norm $n$ is represented in the plane as integer points on the hyperbola $x^{2}-y^{2} d=n$, which has asymptotes

$$
y= \pm \frac{1}{\sqrt{d}} x .
$$

Multiplying an algebraic integer $a+b \sqrt{d}$ by a unit has the effect of translating $a+b \sqrt{d}$ along its hyperbola. Thus, a fundamantal domain for the action of the group of units on the algeberaic integers will lie inside four cones based at the origin, one in each of the four sections of the plane described by the asymptotes.

First we will show that there are only finitely many solutions to (7.17) and (7.19). Our strategy will be to bound each of the integers $w_{i}^{j}$ for $i, j=1,2$. This implies there can be only finitely many $w_{i}$ in a fundamental domain $F$ which satisfy (7.17) and (7.19). Then we will show that this implies there can be only finitely many $h_{i}$ in $F$ which satisfy (7.17) and (7.19) as well. In order to do so, we will use the equivalent set of equations

$$
\begin{gathered}
w_{1}^{1} h_{1}^{1}+w_{2}^{1} h_{2}^{1}=c_{1}=d\left(w_{1}^{2} h_{1}^{2}+w_{2}^{2} h_{2}^{2}\right), \\
w_{1}^{1} h_{1}^{2}+w_{2}^{1} h_{2}^{2}=c_{2}=w_{1}^{2} h_{1}^{1}+w_{2}^{2} h_{2}^{1} .
\end{gathered}
$$

In fact (7.20) and (7.21) can be obtained by a matrix mulitplication

$$
\left(\begin{array}{ll}
w_{1}^{1} & w_{2}^{1} \\
w_{1}^{2} & w_{2}^{2}
\end{array}\right)\left(\begin{array}{ll}
h_{1}^{1} & h_{1}^{2} \\
h_{2}^{1} & h_{2}^{2}
\end{array}\right)=\left(\begin{array}{cc}
c_{1} & c_{2} \\
c_{2} & c_{1} / d
\end{array}\right) .
$$


From (7.22) we see that

$$
\begin{aligned}
\left(\begin{array}{ll}
h_{1}^{1} & h_{1}^{2} \\
h_{2}^{1} & h_{2}^{2}
\end{array}\right) & =\frac{1}{W}\left(\begin{array}{cc}
w_{2}^{2} & -w_{2}^{1} \\
-w_{1}^{2} & w_{1}^{1}
\end{array}\right)\left(\begin{array}{cc}
c_{1} & c_{2} \\
c_{2} & c_{1} / d
\end{array}\right) \\
& =\frac{1}{W}\left(\begin{array}{cc}
c_{1} w_{2}^{2}-c_{2} w_{2}^{1} & c_{2} w_{2}^{2}-\frac{c_{1} w_{2}^{1}}{d} \\
c_{2} w_{1}^{1}-c_{1} w_{1}^{2} & \frac{c_{1} w_{1}^{1}}{d}-c_{2} w_{1}^{2}
\end{array}\right)
\end{aligned}
$$

where $W=w_{1}^{1} w_{2}^{2}-w_{1}^{2} w_{2}^{1} \in \mathbb{Z}$, and $W \mid\left(c_{1}^{2} / d-c_{2}^{2}\right)$.

From (7.23) we obtain the two useful equations

$$
\begin{aligned}
h_{1}^{1}+h_{1}^{2} \sqrt{d} & =\frac{1}{W}\left(\left(c_{1} w_{2}^{2}-c_{2} w_{2}^{1}\right)+\left(c_{2} w_{2}^{2}-\frac{c_{1} w_{2}^{1}}{d}\right) \sqrt{d}\right) \\
& =\frac{1}{W}\left(\left(c_{1}+c_{2} \sqrt{d}\right) w_{2}^{2}-\left(c_{2}+c_{1} / \sqrt{d}\right) w_{2}^{1}\right) \\
& =\frac{1}{W}\left(c_{1}+c_{2} \sqrt{d}\right)\left(w_{2}^{2}-\frac{w_{2}^{1}}{\sqrt{d}}\right)
\end{aligned}
$$

and

$$
\begin{aligned}
h_{2}^{1}+h_{2}^{2} \sqrt{d} & =\frac{1}{W}\left(\left(c_{2} w_{1}^{1}-c_{1} w_{1}^{2}\right)+\left(\frac{c_{1} w_{1}^{1}}{d}-c_{2} w_{1}^{2}\right) \sqrt{d}\right) \\
& =\frac{1}{W}\left(\left(c_{2}+\frac{c_{1}}{\sqrt{d}}\right) w_{1}^{1}-\left(c_{1}+c_{2} \sqrt{d}\right) w_{1}^{2}\right) \\
& =\frac{1}{W}\left(c_{1}+c_{2} \sqrt{d}\right)\left(\frac{w_{1}^{1}}{\sqrt{d}}-w_{1}^{2}\right) .
\end{aligned}
$$

We will break the argument into two cases depending on the sign of the algebraic norm of $w_{1}$ or, equivalently, the sign of $\bar{w}_{1}$. First we will suppose that $\bar{w}_{1}>0$. Since $\bar{w}_{1}>0$ and $w_{1}>0$, we must have that $w_{1}^{1}>0$. We choose a fundamental domain $F$ for the action by units such that the point $(x, y) \in F$ if $-1 / \sqrt{d}<c^{\prime}<y / x<$ $c<1 / \sqrt{d}$, for some constants $c^{\prime}$ and $c$. Since we are assuming $w_{1} \in F$, we have

$$
\frac{-1}{\sqrt{d}}<c^{\prime}<\frac{w_{1}^{2}}{w_{1}^{1}}<c<\frac{1}{\sqrt{d}} .
$$

Since $h_{i}^{1}+h_{i}^{2} \sqrt{d}>0$ for $i=1,2$ and $c_{1}+c_{2} \sqrt{d}>0$, (7.24) and (7.25) imply that

$$
\begin{aligned}
& \frac{1}{W}\left(w_{2}^{2} \sqrt{d}-w_{2}^{1}\right)>0, \\
& \frac{1}{W}\left(w_{1}^{1}-w_{1}^{2} \sqrt{d}\right)>0 .
\end{aligned}
$$

Thus, if $\bar{w}_{1}>0$, (7.28) implies $W>0$ and then (7.27) implies $\bar{w}_{2}<0$. Recall that

$$
W=w_{1}^{1} w_{2}^{2}-w_{1}^{2} w_{2}^{1} .
$$

Equivalently we have

$$
\frac{w_{1}^{2}}{w_{1}^{1}}=\frac{w_{2}^{2}}{w_{2}^{1}}-\frac{W}{w_{2}^{1} w_{1}^{1}} .
$$

Because the integer $W$ divides the integer $c_{1}^{2} / d-c_{2}^{2}$, there are only finitely many possibilities for $W$. Thus, we will think of $W$ as fixed and show that there are only finitely many solutions to (7.30). Since $w_{2}>0$, we have that $w_{2}^{1}>-w_{2}^{2} \sqrt{d}$ and 
since $\bar{w}_{2}<0, w_{2}^{1}<w_{2}^{2} \sqrt{d}$. In particular, $w_{2}^{2}>0$. First suppose $w_{2}^{1}>0$. Then, $w_{2}^{2} / w_{2}^{1}>1 / \sqrt{d}$. Then by (7.26) and (7.30) we have that

$$
\frac{1}{\sqrt{d}}-\frac{W}{w_{1}^{1} w_{2}^{1}}<\frac{w_{1}^{2}}{w_{1}^{1}}<c<\frac{1}{\sqrt{d}}
$$

If either $w_{2}^{1}$ or $w_{1}^{1}$ is too large, we will obtain a contradiction. Thus, $w_{2}^{1}, w_{1}^{1}<k$ for some positive number $k$.

Now suppose that $w_{2}^{1}<0$. Then since $w_{2}>0, \frac{w_{2}^{2}}{w_{2}^{1}}<\frac{-1}{\sqrt{d}}$. Again by (7.26) and (7.30) we have that

$$
\frac{-1}{\sqrt{d}}<c^{\prime}<\frac{w_{1}^{2}}{w_{1}^{1}}<\frac{-1}{\sqrt{d}}-\frac{W}{w_{1}^{1} w_{2}^{1}} .
$$

So if $w_{2}^{1}<0$, it must be bounded below by some negative number $k^{\prime}$. As before, $w_{1}^{1}$ must be bounded above and we will again denote this bound by $k$. We have shown that if $w_{1}^{1}>0, w_{1}^{1}<k$ and $k^{\prime}<w_{2}^{1}<k$. Now, by (7.26), we know that $-k / \sqrt{d}<w_{1}^{2}<k / \sqrt{d}$. Since $w_{2}^{2}>0$, it remains to bound $w_{2}^{2}$ above. From (77.29) we see that

$$
w_{2}^{2}=\frac{W}{w_{1}^{1}}+\frac{w_{1}^{2} w_{2}^{1}}{w_{1}^{1}} .
$$

Since $W, w_{1}^{1}>0, k^{\prime}<w_{2}^{1}<k$, and by (7.26) and (7.31), we have that $w_{2}^{2}<$ $W+\max \left(k / \sqrt{d},-k^{\prime} / \sqrt{d}\right)$.

The case when $\bar{w}_{1}<0$ can be reduced to the previous case in the following way. Choose a unit $u>0$ such that $\bar{u}<0$, and replace $w_{1}$ with $u w_{1}$ and $\bar{w}_{1}$ with $\bar{u} \bar{w}_{1}$. Then $u w_{1}>0$ and $\bar{u} \bar{w}_{1}>0$ and the previous argument applies.

Whether $\bar{w}_{1}<0$ or $\bar{w}_{1}>0$, we have bounded each $w_{i}^{j}$. Since each of these quantities is an integer, there can be only finitely many solutions to (7.29) within a fundamental domain for the action by the group of units for each of the finitely many possibilities for $W$. Now, by (17.24) and (7.25), there can be only finitely many solutions for the quantities $h_{i}^{j}$ as well. Thus, there are only finitely many solutions to (7.17) and (7.19) up to action by units.

Now we prove that (7.18) where $t_{i} \leq w_{i}$ has only finitely many solutions in $O_{d}^{+}$ which lie in a fundamental domain. In fact, we will show that if $0 \leq t_{i} \leq w_{i}$, there can be only finitely many $t_{i}$ in a fundamental domain, and hence only finitely many which satisfy (4.22).

We break the argument into two cases depending on the sign of $\bar{t}_{i}$. Fix $i$ and suppose that $\bar{t}_{i}>0$. Since $t_{i}=t_{i}^{1}+t_{i}^{2} \sqrt{d}$ and $\bar{t}_{i}=t_{i}^{1}-t_{i}^{2} \sqrt{d}, t_{i}, \bar{t}_{i}>0$ implies that $t_{i}^{1}>0$. We have already shown there are only finitely many $w_{i}$ in a fundamental domain which satisfy (7.17) and (7.19). In particular there must be a largest. If $t_{i}$ lies in a fundamental domain and $t_{i} \leq w_{i}$, then $t_{i}$ is bounded above, say by $L$. We have

$$
0 \leq t_{i}^{1}+t_{i}^{2} \sqrt{d} \leq L
$$

and since $t_{i}$ lies in a fundamental domain and $\bar{t}_{i}>0$,

$$
\frac{-1}{\sqrt{d}}<c^{\prime}<\frac{t_{i}^{2}}{t_{i}^{1}}<c<\frac{1}{\sqrt{d}} \text {. }
$$

Equation (7.32) implies that if either $t_{i}^{1}$ or $t_{i}^{2}$ is bounded, then so must the other one be. We will bound $t_{i}^{1}$. In this case, we have already noted that $t_{i}^{1}>0$ so it 
remains to bound $t_{i}^{1}$ from above. Subtracting $t_{i}^{1}$ from both sides of (7.32) and then dividing by the positive quantity $t_{i}^{1} \sqrt{d}$, we can rewrite (7.33) as

$$
\frac{-1}{\sqrt{d}}<c^{\prime}<\frac{t_{i}^{2}}{t_{i}^{1}}<\frac{L}{t_{i}^{1} \sqrt{d}}-\frac{1}{\sqrt{d}}
$$

As $t_{i}^{1} \rightarrow \infty$, the right-hand side of the above tends to $-1 / \sqrt{d}$. This yields a contradiction unless $t_{i}^{1}$ is bounded above.

Next we consider the case where $\bar{t}_{i}<0$. This can be reduced to the previous case by replacing $t_{i}$ with $u t_{i}$ where $u>0$ is a unit such that $\bar{u}<0$. This completes the proof.

\section{ACKNOWLEDGMENT}

I would like to acknowledge and thank A. Eskin for guiding me throughout this project with his tremendous mathematical insight and knowledge. In particular, I wish to thank him for his key observation of the usefulness of the Kenyon and Smillie $J$-invariant which led to so many of the ideas in this paper, including Property X, and for suggesting the original problem from which this paper grew. Furthermore, I would like to thank H. Masur and A. Zorich for many helpful mathematical conversations.

\section{REFERENCES}

[A] P. Arnoux, Echanges d'intervalles et flots sur les surfaces, Ergodic Theory (Sem., Les Planssur-Bex, 1980) (French), 5-38, Monograph. Enseign. Math., 29, Univ. Geneve, Geneva, 1981. MR0609891 (82k:28022)

[GJ] E. Gutkin and C. Judge, Affine mappings of translation surfaces: geometry and arithmetic, Duke Math. J. 103 (2000), no. 2, 191-213. MR1760625 (2001h:37071)

[KS] R. Kenyon and J. Smillie, Billiards on rational angled triangles, Comment. Math. Helv. 75 (2000),no. 1, 65-108. MR1760496 (2001e:37046)

[M1] C. McMullen, Billiards and Teichmuller curves on Hilbert modular surfaces, J. Amer. Math. Soc. 16 (2003), no. 4, no. 2, 857-885. MR.1992827|(2004f:32015)|

[M2] C. McMullen, Dynamics of the $S L(2, \mathbb{R})$ actions in genus 2. Preprint

[Ma] H. Masur, Closed trajectories for quadratic differentials with an application to billiards, Duke Math J. 53 (1986), no. 2, 307-314. MR0850537 (87j:30107)

[R] M. Ratner, On Raghunathan's measure conjecture, Ann.of Math. (2) 134 (1991), no. 3, 545-607. MR 1135878 (93a:22009)

[S] K. Strebel, Quadratic Differentials, Ergebnisse der Mathematik und ihrer Grenzebiete (3) [Results in Mathematics and Related Areas (3)], 5. Springer-Verlag, Berlin, 1984. MR0743423 (86a:30072)

[V1] W. Veech, Teichmuller curves in moduli space, Eisenstein series and an application to triangular billiards, Invent. Math. 97 (1989), no. 3, 553-583. MR.1005006 (91h:58083a)

[V2] W. Veech, Geometric realizations of hyperelliptic curves, Algorithms, fractals, and dynamics (Okayama/Kyoto, 1992), 217-226, Plenum, New York, 1995. MR1402493 (98f:14022)

Department of Mathematics, Cornell University, Ithaca, New York 14853

E-mail address: kcalta@math.cornell.edu 